CONF-9011\%2- -

Busta "

NOV 071990

SAND- $-90-2764 \mathrm{C}$

DE91 002336

\title{
TIME FINITE ELEMENT METHODS FOR LARGE ROTATIONAL DYNAMICS OF MULTIBODY SYSTEMS
}

\author{
F. J. Mello M. Borri S. N. A.tluri \\ Center for Computational Mechanics \\ School of Civil Engineering \\ Georgia Institute of Technology \\ Atlanta, GA 30332
}

\begin{abstract}
Weak formulations in Analytical Dynamics are developed, paralleling the variational methods in elastostatics, and incluring a fundamental yet novel approach for treating constraints (both holonomic and nonholonomic). A general three field appriach is presented, in which the momet tum balance conditions, the compatibility conditions between displacement and velocity, the constitutive relations and the displacement and momentum boundary conditions are all enforced in weak form. A primal, or kinematic formulation is developed from the general form by' enforcing the compatibility conditions and displacement boundary conditions a priori. The conditonal stability of the kinematic formulation is the counterpart of the locking phenomenon in elastostatics and may be avoided, either by reduced order integration, or by utilizing a mixed formulation. Toward this end, a two field mixed formulation
\end{abstract}


is presented, which follows from the general form, when the constitutive relations are satisfied a priori. A general set of the constraint equations are introduced into the kinematic and mixed formulations, using a specific choice of multipliers, which results in modified variational principles. Several simple examples concerning rigid body dynamics are presented.

\section{NOMENCLATURE}

Most of the symbols use in this paper are defined in context and discussed in more detail in our previous work [1,2]. A brief summary is given here.

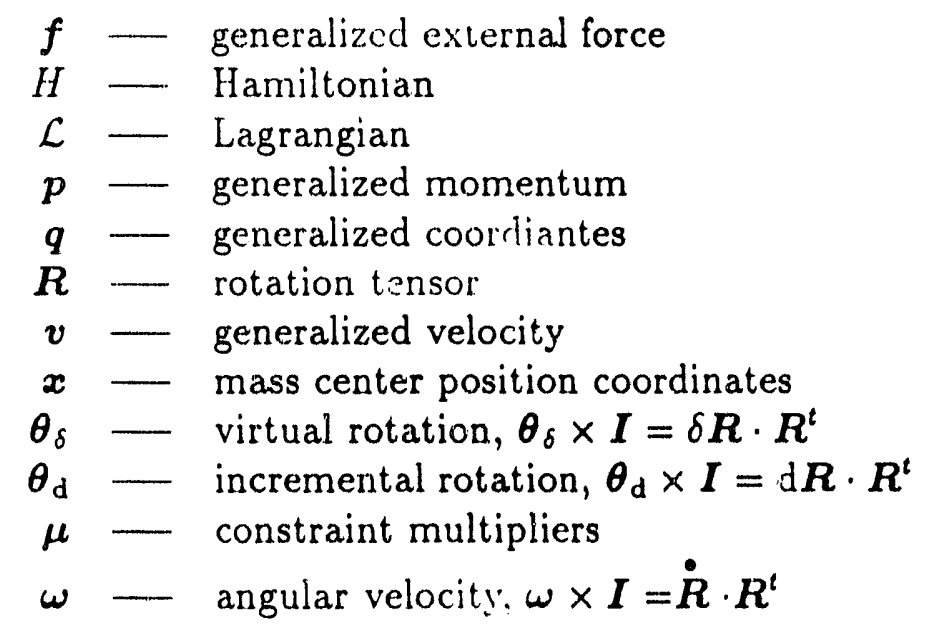

Subscripts on these symbols indicate an association with a particular bndy, or identify boundary quatities.

\section{INTRODUCTION}

Recently there has been a renewed interest in the study of multibody dynamics and its application to a wide variety of engineering problems. The nonlinear equations of motion, in explicit form are guite complex due to the expression for absolute 
acceleration. These complexities are avoided, if a weak formulation is employed. The priciple of virtual work, or Hamilton's principle, is one such weak formulation. There has been much discussion in the literature $[3,4,5]$, concerning Hamilton's principle as a starting poirt for the numerical solution of dynamics problems. Much of this discussion involves the conditions under which Hamilton's principle may be stated as the stationarity condition of a scalar functional. Due to the unsymmetric character of initial value problems, the governing equations are not expressible as such a condition. This fact in no way $i$ for initial value problems. In fact, drawing on the mature literature concerning variational methors in solid mechanics, very general weak forms can be developed for dynamic systems, the most general buing analogous to a $\mathrm{Hu}$ - Washizu type formulation. The principle of virtual work is ol, able from the general weak form by satisfying displacement compatibility and the displacement boundary conditions $a$ priori. A Hamiltonian, or complementary energy approach, is obtained by satisfying the constitutive relations between momentum and velocity a priori .

In two previous papers [1,2], simple, single rigid body problems were used to demonstrate the validity of the primal arıci mixed formulations, for both holonomic and nonholonomic constraints. These example problems, which have either exact solutions, or well known bil: ar. have shown the primal and mixed forins in be accurate numerical methods. In this paper, the highlights of the previous work will be briefly sketched out. We will then discuss the linearization of three common constraints, which may be used to construct multibody systems.

The formation and assembly of the tangent matrices and residual vectors for each body may be carried out without regard for the adjacent bodies, or the characteristics of any joints. In this sense, the multibody problem is no more difficult than the 
single body case. The expressions for constraint residual vectors and tangent matices developed here are combined with those developed in [1], for unconstrained rigid bodies and several multibody examples are solved. We will assess the behavior of the method by considering the two bar system shown in Figure 1. Bar 1 is connected by means of a spherical joint to the fixed datum. Bar 2 is connected to bar 1 by one of the three joint types (spherical, universal or revolute). The results for this simple system, can be juclged intuitively.

Finally, the two dimesional case of a fifteen bar linkage, treated by Kamman and Huston [6], is cosidered for comparison.

One practical complication of multibody problems, as compared with the single body case, is the data structure. In the multibody dynamics literature, there are many references to the Wittenberg - Roberson formalism [7]. The idea of a directed graph, or node to datum array, is an important part of this formalism, since it defines the path from any given body in the system to the reference body. Clearly, this is central to formulating equations of motion in relative joint coordinates. In the relative joint coordinate approach, the position of a body is described in terms of the position of its master (the previous body in the node to datum array). The position of the slave body is completely described, relative to its master, by $n$ coordinates, where $n$ is the number of degrees of freedom allowed by the connection between the bodies. This process results in the minimum number of equations, but is not well suited for systems with closed loops. Relative joint coordinates are typically applied to systems with open chain, or lace configurations. Formulating equations of motion for closed loop systems by relative joint coordinates, requires releasing a sufficient number of connections, in order to form a tree configuration, and then imposing the loop closure conditions as constraints. 
The approach taken here accounts for all constraints by the use of multipliers. Six degrees of freedom are added to the number of global degrees of freedom for each body of the systern. Treating the constrainis individually, their tangent matrices and residual vectors may be calculated and assembled. For each joint, there is a multiplier corresponding to each constrained degree of freedom. Consequently, the system of equations which must be solved is much larger than in the relative joint coordinate approach. The anticipated benefit of this method is, that in a parallel computing environment, all of the matrix formation and assembly for the bodies, and the connections, may be done concurrently. The resulting system of equations is highly structured and very sparse. A diagram showing this structure is presented in Figure 2. This system may then be solved using techniques which exploit the

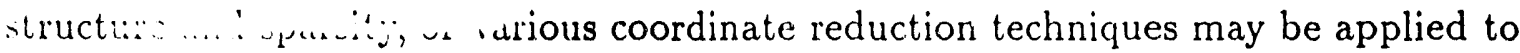
the assembled system of equations [8-12].

\section{WEAK FORMULATIONS IN ANALYTICAL DYNAMICS}

Let us consider a dynamical system with generalized coordinates $\boldsymbol{q}$, acted upon by a generalized exterrial load $f$. We denote the Lagrangian of the system by $\mathcal{L}$, regarded as a function of the coordinates, $\boldsymbol{q}$, the velocities $\boldsymbol{v}$ and time $t$.

The equations of motion:

$$
f+\frac{\partial \mathcal{L}}{\partial \boldsymbol{q}}-\dot{p}=0
$$

the conditions oi compatibility and the constitutive relations:

$$
v=\dot{q} \quad p=\frac{\partial \mathcal{L}}{\partial v}
$$


the displacement and momentum boundary conditions:

$$
\boldsymbol{q}\left(t_{k}\right)=q_{k} \quad \boldsymbol{p}\left(t_{k}\right)=\boldsymbol{p}_{k} \quad k=1,2
$$

can be combined in the following weak form:

$$
\begin{gathered}
\int_{t_{1}}^{t_{2}}\left\{\left(\boldsymbol{f}+\frac{\partial \mathcal{L}}{\partial \boldsymbol{q}}-\dot{\boldsymbol{p}}\right) \cdot \delta \boldsymbol{q}-(\boldsymbol{v}-\dot{\boldsymbol{q}}) \cdot \delta \boldsymbol{p}-\left(\boldsymbol{p}-\frac{\partial \mathcal{L}}{\partial \boldsymbol{v}}\right) \cdot \delta \boldsymbol{v}\right\} \mathrm{d} t= \\
{\left[\left(\boldsymbol{p}\left(t_{2}\right)-\boldsymbol{p}_{2}\right) \cdot \delta \boldsymbol{q}\left(t_{2}\right)-\left(\boldsymbol{q}\left(t_{2}\right)-\boldsymbol{q}_{2}\right) \cdot \delta \boldsymbol{p}\left(t_{2}\right)\right]-} \\
{\left[\left(\boldsymbol{p}\left(t_{1}\right)-\boldsymbol{p}_{1}\right) \cdot \delta \boldsymbol{q}\left(t_{1}\right)-\left(\boldsymbol{q}\left(t_{1}\right)-\boldsymbol{q}_{1}\right) \cdot \delta \boldsymbol{p}\left(t_{1}\right)\right]}
\end{gathered}
$$

which has no subsidiary conditions. Integrating by parts, the terms explicitly involving time derivatives, $\mathrm{Eq}_{\mathrm{q}}(2.4)$ may be simplified as:

$$
\int_{t_{1}}^{t_{2}}\{\delta(\mathcal{L}-\boldsymbol{p} \cdot \boldsymbol{v})+\boldsymbol{f} \cdot \delta \boldsymbol{q}+\delta \dot{\boldsymbol{q}} \cdot \boldsymbol{p}-\delta \dot{\boldsymbol{p}} \cdot \boldsymbol{q}\} \mathrm{d} t=\left[\boldsymbol{p}_{b} \cdot \delta \boldsymbol{q}-\boldsymbol{q}_{b} \cdot \delta \boldsymbol{p}\right]_{t_{1}}^{t_{2}}
$$

where $b=1$ for $t=t_{1}$ and $b=2$ for $t=t_{2}$. This result is analogous to the Hu-Washizu (general 3 field) form [13]. Each of the variables, $\boldsymbol{q}, \boldsymbol{v}$, and $\boldsymbol{p}$, appear as independent fields and have no continuity requirements at the time boundaries. The test functions $\delta \boldsymbol{p}$ and $\delta \boldsymbol{q}$, however must be differentiable on the interval and continuous at the boundary.

The Principle of Virtual Work is obtained from the three field form when the compatibility conditions and displacement boundary conditions are satisfied $a$ priori. Moreover, the momentum field may be eliminated from the domain intcgral assuming the compatibility conditions are also satisfied for the test functions, i.e.: $\delta \boldsymbol{v}=\delta \dot{\boldsymbol{q}}$. In this way we obtain:

$$
\int_{t_{1}}^{t_{2}}(\delta \mathcal{L}+\boldsymbol{f} \cdot \delta \boldsymbol{q}) \mathrm{d} t=\left.\boldsymbol{p}_{b} \cdot \delta \boldsymbol{q}\right|_{t_{1}} ^{t_{2}}
$$

This expression corresponds to Hamilton's principle in configuration space, having only one independent field un unc uvinain $(y)$. Equation (2.6) is reierred to as the 
primal, or kinematic form of Hamilton's principle, and has been successfully applied to mechanical svstems and periodic problems [14,15].

As with the locking phenomenon, which is well known in elasto-statics of volumetrically rigid media or shear rigid thin plates, typical of kinematic formulations, the kinematic form of Hamilton's principle is conditionally stable [2]. We can avoid the resulting restriction on the time step, either through selective reduced integration, or by utilizing a mixed formulation. In fact, if we choose to satisfy the constitutive relations a priori, we may eliminate the velocity as a function of the momentum, and a two field mixed form is obtained. This is accomplished through a Legendre transformation, introducing the Hamiltonian function, $H(\boldsymbol{p}, \boldsymbol{q}, t)=\boldsymbol{p} \cdot \boldsymbol{v}-\mathcal{L}(\boldsymbol{v}, \boldsymbol{q}, t)$, in which $\boldsymbol{v}=\boldsymbol{v}(\boldsymbol{p}, \boldsymbol{q}, t)$ is understıod. Then the two field mixed form involving $\boldsymbol{p}$ and $q$ n::y be written as:

$$
\int_{t_{1}}^{t_{2}}\{-\delta H+\boldsymbol{f} \cdot \delta \boldsymbol{q}+\delta \dot{\boldsymbol{q}} \cdot \boldsymbol{p}-\delta \dot{\boldsymbol{p}} \cdot \boldsymbol{q}\} \mathrm{d} t=\left[\boldsymbol{p}_{b} \cdot \delta \boldsymbol{q}-\boldsymbol{q}_{b} \cdot \delta \boldsymbol{p}\right]_{t_{1}}^{t_{2}}
$$

In this form $\boldsymbol{p}$ and $\boldsymbol{q}$ are treated as coordinates in the phase space, and are not required to be differentiable. It is important to note that since the test and the trial functions have different cintinuity requirements, the test functions $\delta \boldsymbol{p}$ and $\delta \boldsymbol{q}$ are properly understood to be weighting functions, independent of the variations of $\boldsymbol{p}$ and $\boldsymbol{q}$. The numerical implementation of this mixed form is unconditionaliy stable. The stability behavior of the primal and mixed forms is illustrated in a previous paper $[2]$.

\section{WEAK FORM OF CONSTRAINTS}

For the purposes of illustration, we consider only the kinematic and two field mixed forms, and the class of constraints which can be expressed as:

$$
\boldsymbol{\psi}(\dot{\boldsymbol{q}}, \boldsymbol{q}, t)=\mathbf{A}(\boldsymbol{q}, t) \cdot \dot{\boldsymbol{q}}+\boldsymbol{a}(\boldsymbol{q}, t)=0
$$


If calculating the work of constraint forces is to be avoided, the following constraint on the virtual displacements must be enforced.

$$
\mathrm{A} \cdot \delta \boldsymbol{q}=0
$$

Equation (3.1) can be either nonholomonic, or the time derivative of a holonomic constraint. In order to enforce Eq.(3.8) and Eq.(3.9), we cast them in weak form, with a convenient choice of test functions. Let $\boldsymbol{\mu}$ be the Lagrange multipliers. We then weight Eq.(3.8) with the variation $\delta \boldsymbol{\mu}$ and Eq.(3.9) with the time derivative $\dot{\boldsymbol{\mu}}$, obtaining:

$$
\int_{t_{1}}^{t_{2}}\left(\delta \boldsymbol{\mu} \cdot \psi^{\prime \cdot}-\dot{\boldsymbol{\mu}} \cdot \frac{\partial \psi}{\partial \dot{\boldsymbol{q}}} \cdot \delta \boldsymbol{q}\right) \mathrm{d} t=0
$$

The benefit of this form is that it allows an integration by parts that reduces the continuity requirements for the Lagrangian multipliers. Combining Eq.(3.10) with the kinematic form, Eq.(2.6), we obtain a modified form:

$$
\int_{t_{1}}^{t_{2}}(\delta \overline{\mathcal{L}}+\delta \boldsymbol{q} \cdot \overline{\boldsymbol{f}}) \mathrm{d} t=\left.\delta \boldsymbol{q} \cdot \overline{\boldsymbol{p}}_{b}\right|_{t_{1}} ^{t_{2}}
$$

where:

$$
\overline{\mathcal{L}}=\mathcal{L}+\boldsymbol{\mu} \cdot \psi \quad \bar{p}=\boldsymbol{p}+\boldsymbol{\mu} \cdot \frac{\partial \psi}{\partial \boldsymbol{q}} \quad \bar{f}=f+f_{c}
$$

and $f_{c}=\boldsymbol{\mu} \cdot\left(\frac{\mathrm{d}}{\mathrm{dt}} \frac{\partial \boldsymbol{\psi}}{\partial \boldsymbol{q}}-\frac{\partial \boldsymbol{\psi}}{\partial \boldsymbol{q}}\right)$. The Eq.(3.12) is the modified Harnilton's principle for the constrained systems and $\overline{\mathcal{L}}, \overline{\boldsymbol{p}}, \overline{\boldsymbol{f}}$ are respectively the modified Lagrangian function, the modified generalized mor.enta and the external forces modified by the reactions due to the nonholonomic constraints [9]. The constraint reactions, $f_{c}$, are just the weighted integrability conditions of the constraint equations.

It is interesting to note that $\bar{p}$ are the generalized momenta associated with the modified Lagrangian. In fact, it can easily be seen that $\overline{\boldsymbol{p}}=\frac{\partial \overline{\mathcal{L}}}{\partial \dot{\boldsymbol{q}}}$. We can thrn define the modified Hamiltonian function as, $\bar{H}=\overline{\boldsymbol{p}} \cdot \dot{\boldsymbol{q}}-\overline{\mathcal{L}}$ and obtain a mixed form, 
following the procedure discussed in the preceding section:

$$
\int_{t_{1}}^{t_{2}}(-\delta \bar{H}+\delta \boldsymbol{q} \cdot \overline{\boldsymbol{f}}+\delta \dot{\boldsymbol{q}} \cdot \dot{\boldsymbol{p}}-\delta \dot{\overline{\boldsymbol{p}}} \cdot \boldsymbol{q}) \mathrm{d} t=\left.\left(\delta \boldsymbol{q} \cdot \overline{\boldsymbol{p}}_{b}-\delta \overline{\boldsymbol{p}} \cdot \boldsymbol{q}_{b}\right)\right|_{t_{1}} ^{t_{2}}
$$

The modified momenta $\overline{\boldsymbol{p}}$, the generalized coordinates $\boldsymbol{q}$, and the multipliers $\boldsymbol{\mu}$, are the independent fields in this formulation. The true momenta may be recovered from the modified momenta by a projection.

In the numerical examples that follow, we will make use of three common constraint types; the spherical, universal and revolute joints. These are shown in Figures 3,4 and 5 , respectively.

\section{Spherical Joint}

The spherical joint constraint is closely related to the suspension point constraint for the top, which was considered in [2]. The difference is, that the connection point of the spherical joint is not fixed. Figure 3 shows a spherical joint. The location of the connection point, relative to the mass centers of the two connected bodies, is described by position vectors $\rho_{1}$ and $\rho_{2}$, respectively. The constraint, which must be satisfied in order for the joint to stay connected, is:

$$
\dot{x}_{1}-\rho_{1} \times \omega_{1}=\dot{x}_{2}-\rho_{2} \times \omega_{2}
$$

The consulaum on virtl. ".splacement is then:

$$
\delta x_{1}-\rho_{1} \times \theta_{\delta 1}=\delta x_{2}-\rho_{2} \times \theta_{\delta 2}
$$

Following the procedure outline above, the residual vector and tangent matrix for the constraint are calculated. The combined weak form for each side of the constraint equation is:

$$
\int_{t_{1}}^{i_{2}} \delta \boldsymbol{\mu} \cdot(\dot{\boldsymbol{x}}-\boldsymbol{\rho} \times \omega)-\dot{\boldsymbol{\mu}} \cdot\left(\delta \boldsymbol{x}-\boldsymbol{\rho} \times \theta_{\delta}\right) \mathrm{u} \iota
$$


Subscripts are omitted, but it is understood this expression must be evaluated for each of the bodies attached at the joint. The difference in these results is the residual vector. Integrating by parts the term involving $\dot{\mu}$, leads to:

$$
\int_{t_{1}}^{t_{2}} \delta \mu \cdot(\dot{x}-\rho \times \omega)+\boldsymbol{\mu} \cdot\left(\delta \dot{\boldsymbol{x}}-\rho \times \dot{\theta}_{\delta}-\dot{\rho} \times \theta_{\delta}\right) \mathrm{d} t=\left.\boldsymbol{\mu} \cdot\left(\delta \boldsymbol{x}-\rho \times \theta_{\delta}\right)\right|_{t_{1}} ^{t_{2}}
$$

The terms under the integral in Eq.(3.10) constitute the residual vector.

$$
\{(\dot{x}-\rho \times \omega), \mu,(\rho \times \mu), 0,(\stackrel{\rho}{\rho} \times \mu)\}
$$

where the organization of the test functions is $\left(\delta \boldsymbol{\mu}, \dot{\delta} \boldsymbol{x}, \dot{\theta}_{\delta}, \delta \boldsymbol{x}, \theta_{\delta}\right)$. It should be noted that $t$ lin test functions are expressed in "quasi-coordinates". Since the incremental solution process must use true coordinates for the trial functions, the associated tangent matrix will be unsymmetric. The linearization process is more easily accomplished by using "quasi-coordinates" and later tranforming the matrix to involve true coordinates, as discussed in $[1,2]$.

The tangent matrix for this constraint is obtained by linearizing the residual vector. The following identities are discussed in Appendix A of [1], and are repeated here for convenience.

$$
\begin{gathered}
\mathrm{d} \rho=\theta_{\mathrm{d}} \times \rho=-\rho \times \theta_{\mathrm{d}} \\
\dot{\rho}=\omega \times \rho \\
\mathrm{d} \dot{\rho}=\dot{\theta}_{\mathrm{d}} \times \rho+\theta_{\mathrm{d}} \times \dot{\rho}=-\rho \times \dot{\theta}_{\mathrm{d}}-\dot{\rho} \times \theta_{\mathrm{d}} \\
\mathrm{d} \boldsymbol{\omega}=\dot{\theta}_{\mathrm{d}}-\boldsymbol{\omega} \times \theta_{\mathrm{d}}
\end{gathered}
$$

With these relations in mind, we may perform the linearization. Linearizing the first group of terms $:_{n}$ Eq.(3.11), corresponding to the test functions $\delta \mu$, leads to:

$$
\delta \boldsymbol{\mu} \cdot(\mathrm{d} \dot{\boldsymbol{x}}-\mathrm{d} \boldsymbol{\rho} \times \boldsymbol{\omega}-\boldsymbol{\rho} \times \mathrm{d} \boldsymbol{\omega})
$$


which, in view of the above identities, simplifies to:

$$
\delta \mu \cdot[\boldsymbol{I},(\rho \times I),(\rho \times \omega) \times I] \cdot\left\{\begin{array}{c}
\mathrm{d} \dot{x} \\
\dot{\theta_{\mathrm{d}}} \\
\theta_{\mathrm{d}}
\end{array}\right\}
$$

Similarly, the second group of terms in the residual vector, corresponding to the test functions $\delta \dot{\boldsymbol{x}}$, lead to:

$$
\delta \dot{\boldsymbol{x}} \cdot \boldsymbol{I} \cdot \mathrm{d} \boldsymbol{\mu}
$$

The terms involving the test functions $\dot{\theta}_{\delta}$ linearize as:

$$
\dot{\theta_{\delta}} \cdot(\mathrm{d} \rho \times \mu+\rho \times \mathrm{d} \mu)
$$

which reduces to:

$$
\dot{\theta}_{\delta} \cdot[\rho \times I, \mu \times \rho \times I] \cdot\left\{\begin{array}{c}
\mathrm{d} \boldsymbol{\mu} \\
\theta_{\mathrm{d}}
\end{array}\right\}
$$

Finally, the last group of terms in the residual vector leads to:

$$
\theta_{\delta} \cdot(\mathrm{d} \dot{\rho} \times \mu+\dot{\rho} \times \mathrm{d} \mu)
$$

which may be written as:

$$
\theta_{\delta} \cdot\left[\dot{\rho} \times I, \mu \times \rho \times \dot{\theta}_{\mathrm{d}}, \mu \times \dot{\rho} \times I\right] \cdot\left\{\begin{array}{c}
\mathrm{d} \mu \\
\dot{\theta}_{\mathrm{d}} \\
\theta_{\mathrm{d}}
\end{array}\right\}
$$

Combining these relations provides the tangent matrix for the constraint.

$$
\mathcal{T}=\left[\begin{array}{ccccc}
0 & \boldsymbol{I} & -\boldsymbol{\rho} \times \boldsymbol{I} & 0 & -\dot{\rho} \times \boldsymbol{I} \\
\boldsymbol{I} & 0 & 0 & 0 & 0 \\
\rho \times I & 0 & 0 & 0 & \boldsymbol{\mu} \times \boldsymbol{\rho} \times \boldsymbol{I} \\
0 & 0 & 0 & 0 & 0 \\
\dot{\rho} \times I & 0 & \boldsymbol{\mu} \times \boldsymbol{\rho} \times \boldsymbol{I} & 0 & \boldsymbol{\mu} \times \dot{\rho} \times \boldsymbol{I}
\end{array}\right]
$$


Lastly, this tangent matrix must be transformed so that the linearization involves increments of true coordinates. This transformation is discussed in [2] and is not repeated here.

The spherical joint forms the basis for the other two joints considered below.

\section{Universal Joint}

Next, the universal joint depicted in Figure 4 is considered. The joint consists of a spherical joint, with the additional constraint that the unit vectors $i$ and $j$, shown in the figure, remain perpendicular ihroughout the rnotion. These vectors are fixed in bodies 1 and 2, respectively. This constraint can be written concisely as:

$$
\left(R_{1} \cdot i\right) \cdot\left(R_{2} \cdot j\right)=0
$$

where $\boldsymbol{R}_{1}$ and $\boldsymbol{R}_{2}$, denote the rotations of bodies 1 and 2 , respectively. Using the definitions of the angular velocities of bodies 1 and 2 , this constraint may be expressed in differential form as:

$$
\left[\left(\omega_{1} \times I\right) \cdot R_{1} \cdot i\right] \cdot\left[R_{2} \cdot j\right]+\left[R_{1} \cdot i\right] \cdot\left[\left(\omega_{2} \times I\right) \cdot R_{2} \cdot j\right]=0
$$

Letting $\boldsymbol{i}^{\prime}=\boldsymbol{R}_{1} \cdot \boldsymbol{i}$ and $\boldsymbol{j}^{\prime}=\boldsymbol{R}_{\mathbf{2}} \cdot \boldsymbol{j}$, Eq.(3.32) takes ox the simpler form:

$$
\left(i^{\prime} \times j^{\prime}\right) \cdot \omega_{1}-\left(i^{\prime} \times j^{\prime}\right) \cdot \omega_{2}=0
$$

The constraint on virtual displacements is then:

$$
\left(i^{\prime} \times j^{\prime}\right) \cdot \theta_{\delta 1}-\left(i^{\prime} \times j^{\prime}\right) \cdot \theta_{\delta 2}=0
$$

Again, the constraint Eq.(3.33) is weighted with the variation of the multiplier $\delta \mu$, while Eq.(3.34) is weighted with the time derivative $\dot{\mu}$. Combining these weak forms and performing the integration by parts, leads to:

$$
\begin{aligned}
\int_{t_{1}}^{t_{2}} \delta \mu \cdot \boldsymbol{A} \cdot\left(\omega_{1}, \omega_{2}\right) & +\frac{\mathrm{d}}{\mathrm{d} t}\left(\theta_{\delta 1}, \theta_{\delta 2}\right) \cdot \boldsymbol{A}^{t} \cdot, \cdot\left(\theta_{s_{1}}, \theta_{\delta 2}\right) \cdot \dot{\boldsymbol{A}}^{t} \cdot \mu \mathrm{d} t \\
& =\left.\left(\theta_{\delta_{1}}, \theta_{\delta 2}\right) \cdot \boldsymbol{A}^{t} \cdot \mu\right|_{t_{1}} ^{t_{2}}
\end{aligned}
$$


where $A=\left(i^{\prime} \times j^{\prime},-i^{\prime} \times j^{\prime}\right)$.

If the tes: funciions are arranged as $\left(\delta \mu, \dot{\theta_{\delta 1}}, \theta_{\delta 1}, \dot{\theta_{\delta 2}}, \theta_{\delta 2}\right)$, then the residual vector may be written as:

$$
\left\{\begin{array}{c}
\left(i^{\prime} \times j^{\prime}\right) \cdot \omega_{1}-\left(i^{\prime} \times j^{\prime}\right) \cdot \omega_{2} \\
\left(i^{\prime} \times j^{\prime}\right) \mu \\
\left(\left(\omega_{1} \times i^{\prime}\right) \times j^{\prime}+i^{\prime} \times \omega_{2} \times j^{\prime}\right) \mu \\
-\left(i^{\prime} \times j^{\prime}\right) \mu \\
\left(\left(\omega_{2} \times j^{\prime}\right) \times i^{\prime}+j^{\prime} \times \omega_{1} \times i^{\prime}\right) \mu
\end{array}\right\}
$$

The fact that $i$ is fixed with respect to body 1 , and $j$ is fixed relative to body 2 , has been used to express $i^{\prime}=\omega_{1} \times i^{\prime}$ and $j^{\prime}=\omega_{2} \times j^{\prime}$. Similarly, the virtual changes in $i$ and $j^{\prime}$, are given as $\delta i^{\prime}=\theta_{\delta 1} \times i^{\prime}$ and $\delta j^{\prime}=\theta_{\delta 2} \times j^{\prime}$, respectively. The linearization of this residual vector is tedious, but straightforward. The resulting tangent matrix may be verified to be:

$\left[\begin{array}{c|c|c|c|c}0 & \mu\left(j^{\prime} \times i^{\prime} \times I\right) & 0 & -\mu\left(\boldsymbol{i}^{\prime} \times j^{\prime} \times I\right) & i^{\prime} \times j^{\prime} \\ \hline \mu\left(j^{\prime} \times \boldsymbol{i}^{\prime} \times I\right) & \mathcal{C}_{1} & -\mu\left(\boldsymbol{i}^{\prime} \times j^{\prime} \times I\right) & \mathcal{C}_{2} & \mathcal{C}_{3} \\ \hline 0 & -\mu\left(j^{\prime} \times i^{\prime} \times I\right) & 0 & \mu\left(\boldsymbol{i}^{\prime} \times j^{\prime} \times \boldsymbol{I}\right) & -\boldsymbol{i}^{\prime} \times j^{\prime} \\ \hline-\mu\left(j^{\prime} \times i^{\prime} \times \boldsymbol{I}\right) & -\mathcal{C}_{1} & \mu\left(\boldsymbol{i}^{\prime} \times j^{\prime} \times I\right) & -\mathcal{C}_{2} & \mathcal{C}_{4} \\ \hline-\boldsymbol{i}^{\prime} \times j^{\prime} & \mathcal{C}_{5} & -\boldsymbol{i}^{\prime} \times j^{\prime} & \mathcal{C}_{6} & 0\end{array}\right]$

where:

$$
\mathcal{C}_{1}=\left[\begin{array}{c}
j^{\prime} \times \omega_{1} \times i^{\prime} \times I \\
-j^{\prime} \times i^{\prime} \times \omega_{1} \times I \\
+\left(\omega_{2} \times j^{\prime}\right) \times i^{\prime} \times I
\end{array}\right]
$$




$$
\begin{array}{r}
\mathcal{C}_{2}=\left[\begin{array}{r}
-i^{\prime} \times \omega_{2} \times j^{\prime} \times I \\
+i \times j^{\prime} \times \omega_{2} \times I \\
-\left(\omega_{1} \times i^{\prime}\right) \times j^{\prime} \times I
\end{array}\right] \\
\mathcal{C}_{3}=\left[\begin{array}{c}
\left(\omega_{1} \times i^{\prime}\right) \times j^{\prime}+ \\
i^{\prime} \times \omega_{2} \times j^{\prime}
\end{array}\right] \\
\mathcal{C}_{4}=\left[\begin{array}{c}
\left(\omega_{2} \times j^{\prime}\right) \times i^{\prime}+ \\
j^{\prime} \times \omega_{1} \times i^{\prime}
\end{array}\right] \\
\mathcal{C}_{5}=\left[\begin{array}{c}
j^{\prime} \times i^{\prime} \times\left(\omega_{1}-\omega_{2}\right) \\
+\omega_{1} \times i^{\prime} \times j^{\prime}
\end{array}\right]
\end{array}
$$

\section{Revolute Joint}

The last joint type which will be considered here is the revolute joint, depicted in Figure 5. The constraint provided by this joint is that the relative rotation between body 1 and body 2 must be about the axis of the joint. In order to describe the constraint, the vectors $\boldsymbol{i}, \boldsymbol{j}$ and $\boldsymbol{k}$ shown in Figure 5, are defined as follows. The vector $i$ is fixed in body 1 , and is the unit vector normal to the plane defined by the hinge axis and the center of mass of body 1 . Vector $j$ is then defined to be a unit vector normal to the plane of the hinge axis and $i$, and is fixed in body 1 . The unit vector along the axis of the hinge is $\boldsymbol{k}$, but will be associated with body 2 . The revolute joint constraint may then be expressed as a sperical joint, with the additional constraints that:

$$
\boldsymbol{k} \cdot \boldsymbol{i}=0 \quad \text { and } \quad \boldsymbol{k} \cdot \boldsymbol{j}=0
$$

In differential form, these two constraint equations are:

$$
\begin{aligned}
& \left(\omega_{2} \times k\right) \cdot i+k \cdot\left(\omega_{1} \times i\right)=0 \\
& \left(\omega_{2} \times k\right) \cdot i+k \cdot\left(\omega_{1} \times i\right)=0
\end{aligned}
$$


which, in matrix form, appears as:

$$
\left[\begin{array}{cc}
i \times k & -i \times k \\
j \times k & -j \times k
\end{array}\right] \cdot\left\{\begin{array}{l}
\omega_{1} \\
\omega_{2}
\end{array}\right\}=0
$$

The matrix in Eq.(3.44) is the $\boldsymbol{A}$ matrix of Eq.(3.8), for this constraint. The weak form of the constraint, after carrying out the integration by parts, is then:

$$
\begin{aligned}
\int_{t_{1}}^{t_{2}} \delta \mu \cdot \mathbf{A} \cdot\left(\omega_{1}, \omega_{2}\right) & +\boldsymbol{\mu} \cdot \dot{\mathbf{A}} \cdot\left(\theta_{\delta 1}, \theta_{\delta 2}\right)+\boldsymbol{\mu} \cdot \mathbf{A} \cdot\left(\dot{\theta}_{\delta 1}, \dot{\theta_{\delta 2}}\right) \mathrm{d} t \\
= & \left.\left(\theta_{\delta 1}, \theta_{\delta 2}\right) \cdot \mathbf{A}^{t} \cdot \boldsymbol{\mu}\right|_{t_{1}^{2}} ^{t_{2}}
\end{aligned}
$$

where the matrix $\dot{A}$ is given by:

$$
\dot{\mathrm{A}}=\left[\begin{array}{cc}
\left(\omega_{1} \times i\right) \times k+i \times \omega_{2} \times k & -\left(\omega_{1} \times i\right) \times k-i \times \omega_{2} \times k \\
\left(\omega_{1} \times j\right) \cdots k+j \times \omega_{2} \times k & -\left(\omega_{1} \times j\right) \times k-j \times \omega_{2} \times k
\end{array}\right]
$$

The residual vector, in this case, may then be written as:

$$
\left\{\begin{array}{c}
\mu_{1}(\boldsymbol{i} \times \boldsymbol{k})+\mu_{2}(j \times k) \\
\mu_{1}\left(\boldsymbol{k} \times \boldsymbol{i} \times\left(\omega_{1}-\omega_{2}\right)\right)+\mu_{2}\left(\boldsymbol{k} \times j \times\left(\omega_{1}-\omega_{2}\right)\right) \\
-\mu_{1}(i \times k)-\mu_{2}(j \times k) \\
-\mu_{1}\left(k \times i \times\left(\omega_{1}-\omega_{2}\right)\right)-\mu_{2}\left(k \times j \times\left(\omega_{1}-\omega_{2}\right)\right) \\
(i \times k) \cdot\left(\omega_{1}-\omega_{2}\right) \\
(j \times k) \cdot\left(\omega_{1}-\omega_{2}\right)
\end{array}\right\}
$$

The organization of the test functionsm is; $\dot{\theta_{\delta 1}}, \theta_{\delta_{1}}, \dot{\theta_{\delta 2}}, \theta_{\delta 2}$ and $\delta \mu$. Again, linearization of this residual vector leads to the tangent matrix for the revolute joint. 


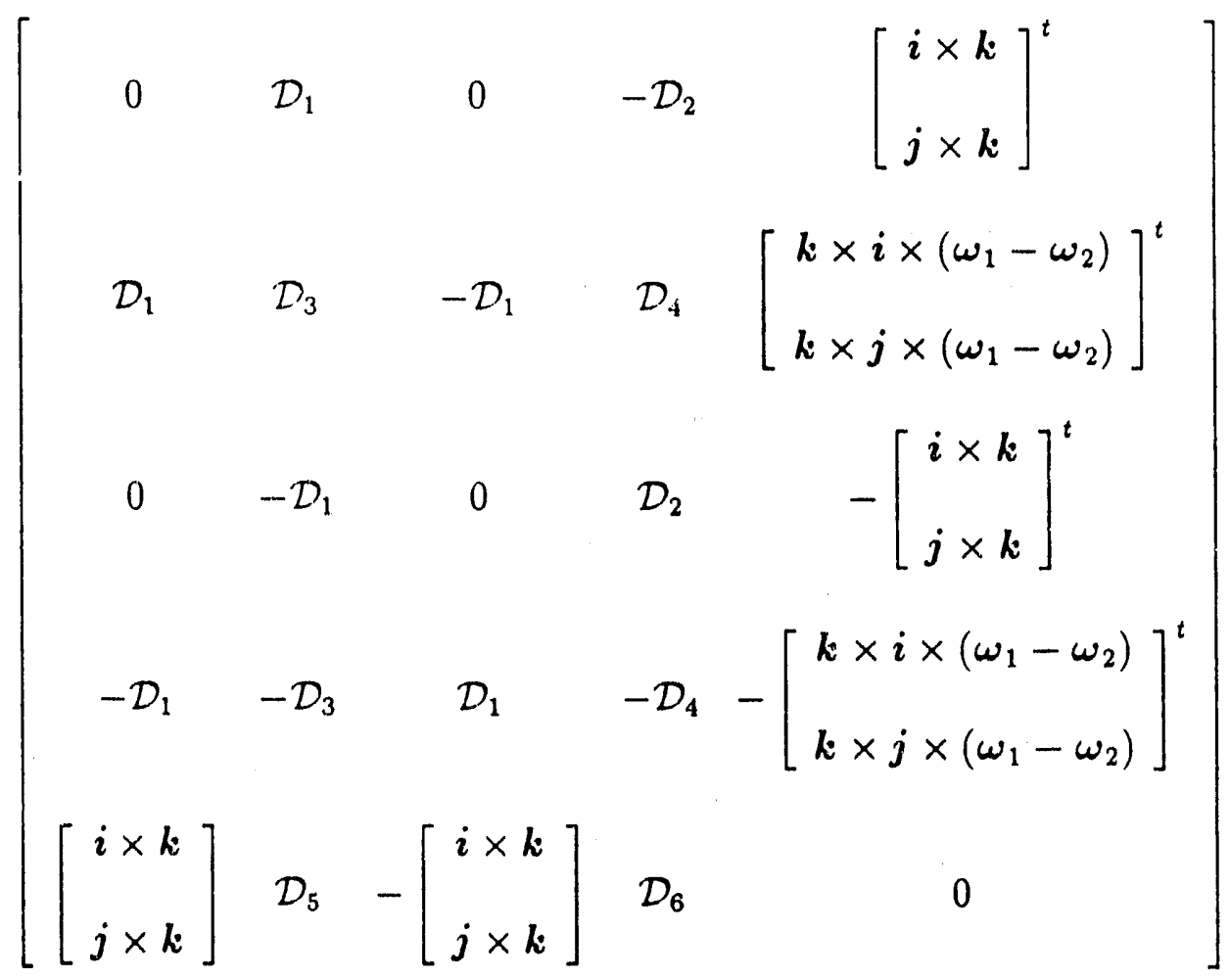

where the submatrices $\mathcal{D}_{1}, \mathcal{D}_{2}, \mathcal{D}_{3} \mathcal{D}_{4}, \mathcal{D}_{5}, \mathcal{D}_{6}$ are given by:

$$
\begin{gathered}
\mathcal{D}_{1}=\left[\begin{array}{c}
\mu_{1}(k \times i \times I) \\
+\mu_{2}(k \times j \times I)
\end{array}\right] \quad \mathcal{D}_{2}=\left[\begin{array}{c}
\mu_{1}(i \times k \times I) \\
+\mu_{2}(j \times k \times I)
\end{array}\right] \\
\mathcal{D}_{3}=\left[\begin{array}{c}
\mu_{1}\left(k \times\left(\omega_{1}-\omega_{2}\right) \times i \times I-k \times i \times \omega_{1} \times I\right) \\
+\mu_{2}\left(k \times\left(\omega_{1}-\omega_{2}\right) \times j \times I-k \times j \times \omega_{1} \times I\right)
\end{array}\right] \\
\mathcal{D}_{4}=\left[\begin{array}{c}
\mu_{1}\left(\left(i \times\left(\omega_{1}-\omega_{2}\right)\right) \times k \times I+k \times i \times \omega_{2} \times I\right) \\
+\mu_{2}\left(\left(j \times\left(\omega_{1}-\omega_{2}\right) \times k \times I+k \times j \times \omega_{2} \times I\right)\right.
\end{array}\right] \\
\mathcal{D}_{5}=\left[\begin{array}{c}
k \times i \times\left(\omega_{1}-\omega_{2}\right) \\
+\omega_{1} \times i \times k \\
k \times j \times\left(\omega_{1}-\omega_{2}\right) \\
+\omega_{1} \times j \times k
\end{array}\right] \quad\left[\begin{array}{c}
i \times k \times\left(\omega_{1}-\omega_{2}\right) \\
-\omega_{2} \times i \times k \\
j \times k \times\left(\omega_{1}-\omega_{2}\right) \\
-\omega_{2} \times j \times k
\end{array}\right]
\end{gathered}
$$




\section{NUMERICAL EXAMPLES}

The primal and mixed forms, for holonomic and nonholonomic constraints, have been verified in our previous work $[1,2]$. Here, we consider several simple numerical examples to illustrate the extension of the approach to multibody systems. Each of the three joints considered in the previous section is verified by a simple two body problem. As an additional check, the degenerate case of a planar linkage is compared with the results presented by Kamman and Huston [6].

For the spherical joint, the procedure for establishing constraint equations is to form and assemble the residual vector and tangent matrix for the first body connected at the joint. Then, the residual vector and tangent matrix are calculated for the second body, and assembled with the opposite sign. So, for each node of the time element, there is a three by twelve constraint matrix, which couples the degrees of freedom of the connected bodies. To test the joint element, the two bar example of Figure 1 has been run, with the problem definition given as:

- Both bars start in a position aligned with the negative y axis.

- The initial velocities are given, in body fixed coordinates, as $\boldsymbol{v}_{1}=(2,0,0)$, $\omega_{1}=(0,0,4)$ and $\boldsymbol{v}_{2}=(6,0,0), \boldsymbol{\omega}_{2}=(0,0,4)$.

- The only external force acting on the bars is gravity.

Results for this case are presented in Figures 6,7 and 8. This problem and the other multibody examples presented, use a three noded primal time finite element. The $\mathrm{XY}$ displacement is shown in Figure 6, while the $\mathrm{YZ}$ and $\mathrm{XZ}$ displacement curves are plotted in Figures 7 and 8, respectively. 
As a further check that the program is predicting correct behavior, the same problem was repeated, with the exception that bar 2 was given a high anguar velocity, along its axis. Figures 9,10 and 11 show, respectively, the XY, YZ and XZ displacement for this case. As expected, bar 2 remains very nearly horizontal throughout the motion.

The universal joint has been tested by solving a similar problem. In this case, bar 1 is started in the vertical position, while bar 2 is started in a horizontal position. This example is started with the following initial conditions:

- Bar 1 is aligned with the negative $\mathrm{z}$ axis, bar 2 is alligned with the negative $y$ axis.

- The initial velocities are given, in body fixed coordinates, as $\boldsymbol{v}_{1}=(0,0,0)$, $\omega_{1}=(0,6,0)$ and $v_{2}=(3,0,0), \omega_{2}=(0,0,6)$.

- The only external force acting on the bars is gravity.

In this situation, one would expect bar 2 to be thrown outward, away from the axis of rotation tending to straighten the linkage. As the bars become more nearly straight, a whirling type motion would result. This is, in fact, the behavior which is observed in the simulation (see Figures 12, 13 and 14).

The revolute joint has been verified in a similar fashion, but the results are not included here. The behavior of the revolute joint is most easily seen by animating the solution.

Finally, we consider the fifteen bar linkage presented by Kamman and Iluston [6]. Equations of motion, developed using Kane's method, are used in their simulation. Since this problem is planar, it docs not demonstrate the accuracy of a three 
dimensional multibody program. However, it does show that the implementation degenerates correctly to the two dimensional case.

The original configuration of the linkage is shown in Figure 15. The linkage consists of fifteen identical bars, connected by spherical joints. Points $\mathrm{B}$ and $\mathrm{C}$ are released, and the linkage is allowed to fall. The shape of the chain is calculated for times between 0 and 1 second. The input data for this problem are as follows:

$$
\begin{aligned}
& \mathrm{M}=.4565 \text { slugs } \quad=\text { Mass } \\
& \mathrm{L}=1.0 \mathrm{ft} . \quad=\text { Length } \\
& \mathrm{I}_{t}=.0308 \text { slug }-\mathrm{ft}^{2}=\text { Transverse Inertia } \\
& \mathrm{I}_{a}=.0001 \text { slug }-\mathrm{ft}^{2}=\text { Axial Iner ùia }
\end{aligned}
$$

Here, the axial inertia is simply set to any nonzero number. The linkage falls, as shown in Figure 16, and the center link reaches a low point at $t=.47$ seconds. At this point, the motion reflects and the center bar starts to move upward. The plint of the motion from $t=.47$ seconds until $t=1$ second is shown in Figure 17. Figure 18, shows a, plot of the vertical displacement of the center bar vs. time. This clearly shows, the reflection point at $\mathrm{t}=.47$, and another point at $\mathrm{t}=.69$, where the bar stops moving up and begins to fall again. These results agree very well with those presented by Kamman and Huston [6].

\section{CONCLUSIONS}

Kinematic and mixed forms for dynamics, derived form a very general framework are presented. Multibody simulations are carried out using finite elements in the time domain. A new approach for incorporating constraints is shown to be an effective and general method of enforcing interbody connections.

The intuitive results obtained for the three dimensional motion of the two bar system. coupled with the results for the planar linkage, verify the presented procedures. 


\section{ACKNOWLEDGEMENTS}

The authors gratefully acknowledge the siinjort of the Air Force Office of Scientific Research.

\section{REFERENCES}

1. M. Borri, F. J. Mello and S. N. Atluri. "Variational Approaches for Dynamics and Time - Finite - Elements: Numerical Studies", Computational Mechanics, to appear

2. M. Borri, F. J. Mello and S. N. Atluri. "Primal and Mixed Forms of Hamilton's Principle for Consträned Rigid Body Systems: Numerical Studies", Computational Mechanics, to appear.

3. C. D. Bailey. "Application of Hamilton's Law to Forced, Damped, Stationary Systems", Journal of Sound and Vibration, Vol. 75, pp. 117-126, 1981.

4. M. Baruch and R. Riff. "Hamilton's Principle, Hamilton's Law, 6" correct formulations", AIAA Journal, Vol. 21, pp. 687-692, 1982.

5. T. E. Simkins. "Finite Element for Initial Value Problems in Dynamics", AIAA Journal, Vol. 13, pp. 1154-1157, 1981.

6. J. W. Kamman and R. L. Huston. "Dynamics of Constrained Multibody Systems", Journal of Applied Mechanics, Vol. 51, pp. S99-903, 1984.

7. J. Wittenburg. "Dynamics of Multibody Sy'stems", International Union of Theoretical and Applied Mechanics, pp. 197-207, 1980. 
8. A. J. Kurdila. "Concurrent Miltiprocessors in Computational Mechanics for Constrained Dynamical Systems", Fin.D. thesis, Georgia Institute of Technology, Dec. 1988.

9. P. E. Nikravesh and E. J. $f^{\prime}$ ıug. "Generalized Coordinate Partioning for Analysis of Mechanical Systems with Nonholonomic Constraints", Journal of Mechanisms, Transmissions, and Automation in Design, September 1983, Vol. 105, pp. 379-384.

10. R. A. Wehage and E. J. Haug. "Generalized Coordinate Partitioning for Dimension Reduction in Analysis of Constrained Dynamic Systems", Journal of Mechanical Design, Vol. 104, pp. 247-255, 1982.

11. R. P. Singh and P. W. Likins. "Singular Value Decomposition for Constrained Dynamical Systems", Journal of Applied Mechanics, 'ol. 52, pp. 943-948, 1985.

12. F. M. L. Amirouche and T. Jai. "Automatic Elimination of the Undetermined Multipliers in Kane's Equations Using a Pseudo Uptriangular Decomposition (PUTD) Method", Computers and Structures, Vol. 27, No. 2, pp 203-21.0, 1987.

13. K. Washizu. Variational Methods in Elasticity and Plasticity, third edition, Pergamon 1980.

14. M. Borri, G. L. Ghiringhelli, M. Lanz, P. Mantegazza and T. Merlini. "Dynamic Response of Mechanical Systems by a Weak Hamilton's Formulation", Computers \& Structures, Vol. 20, pp. 495-508, 1985. 
15. M. Borri, M. Lanz and P. Mantegazza. "Helicopter Rotor Dynamics by Finite Element Time Discretization". L'Aerotecnica Missili e Spazio, Vol. 60, pp. 193$200,1981$.

\section{DISCLAIMER}

This report was prepared as an account of work sponsured by an agency of the United States Government. Neither the United States Government nor any agency thereof, nor any of their employees, makes any warranty, express or implied, or assumes any legal liability or responsibility for the accuracy, completeness, or usefulness of any information, apparatus, product, or process disclosed, or represents that its use would not infringe privately owned rights. Reference herein to any specific commercial product, process, or service by trade name, trademark, manufacturer, or otherwise does not necessarily ionstitute or imply its endorsement, recommendation, or favoring by the United States Government or any agency thereof. The views and opinions of authors expressed herein do not necessarily state or reflect those of the United States Government or any agency thereof. 


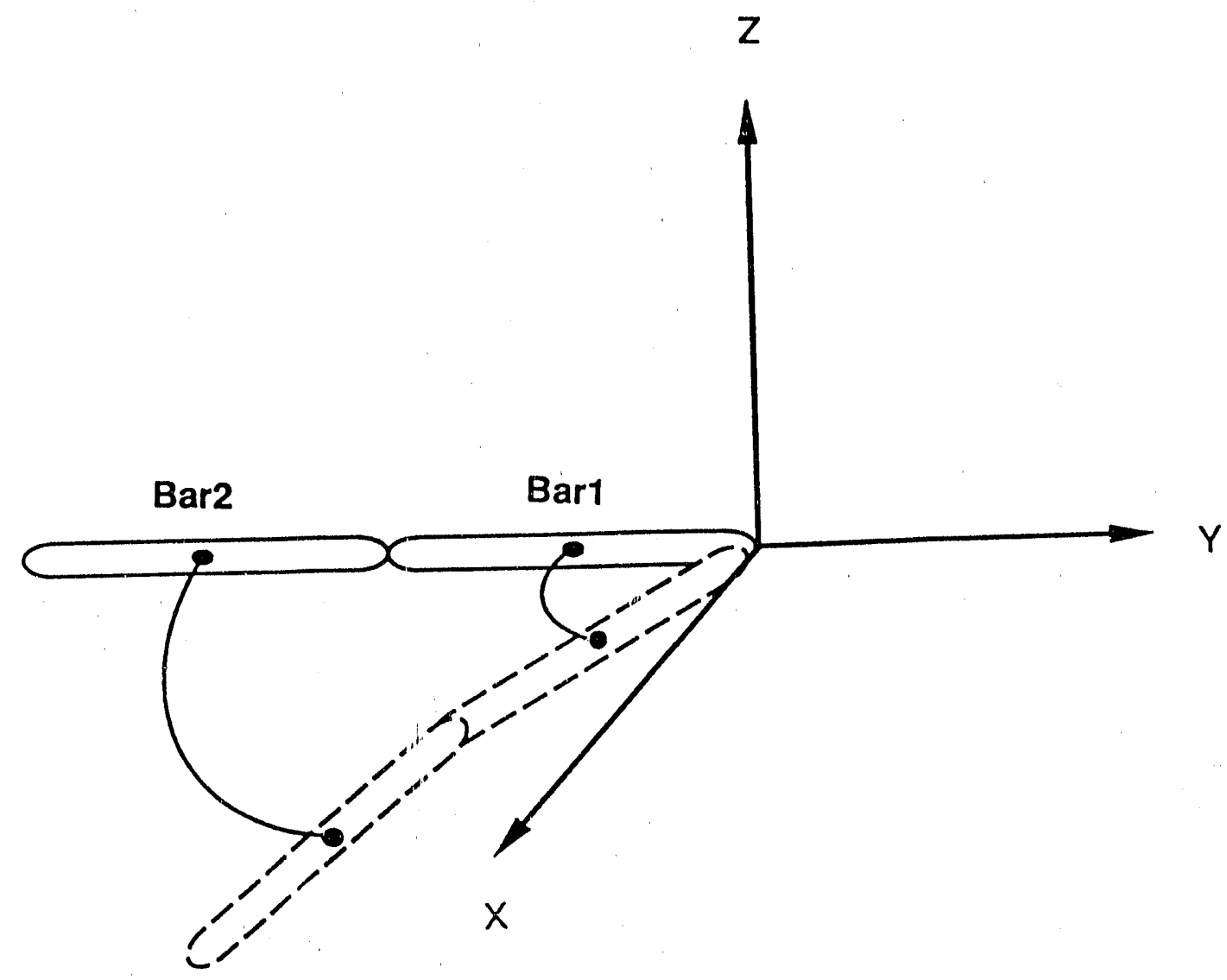

Figure 1: Two Bar Test Problem 


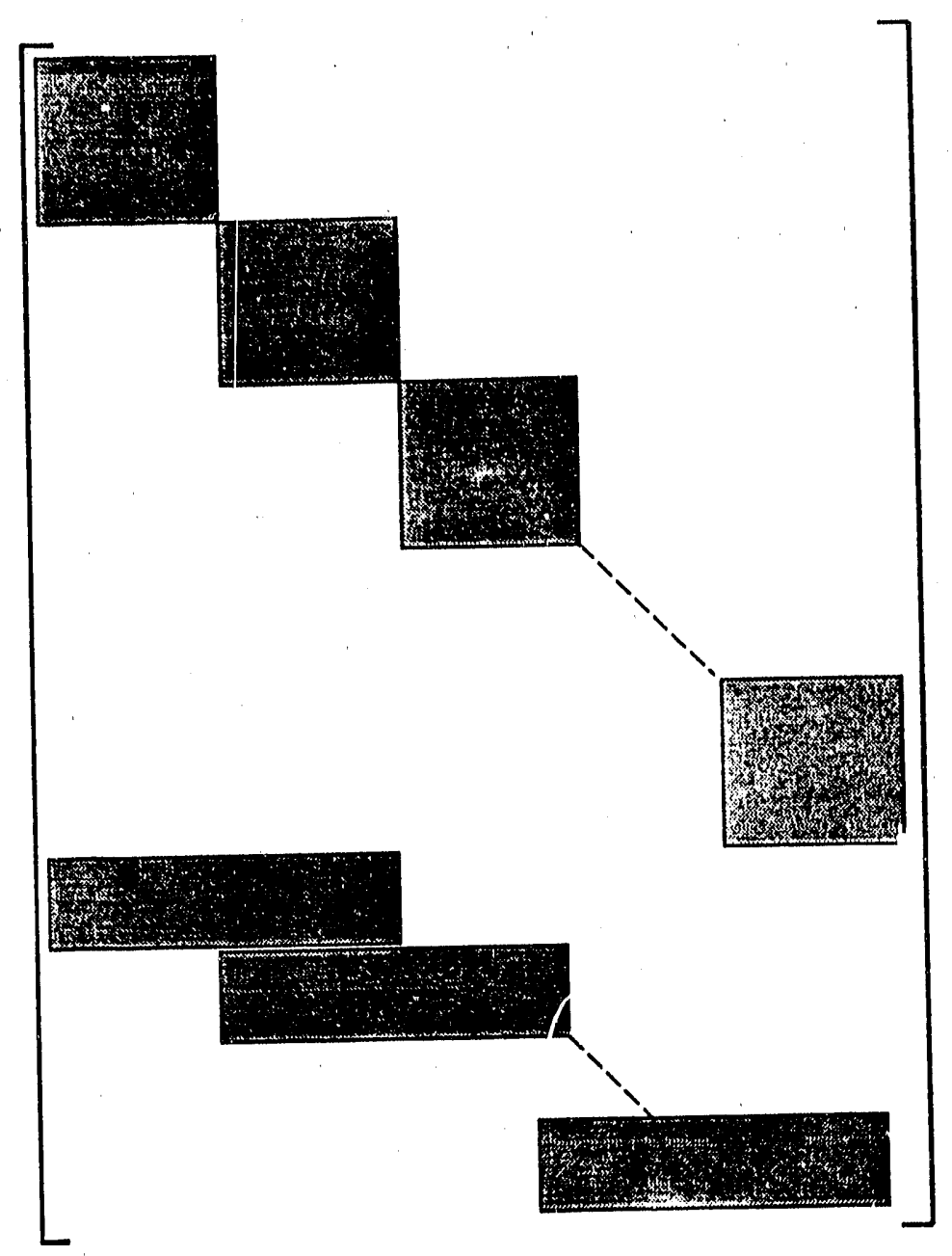

Figure 2: Sparse Matrix Structure for Multibody Problems 


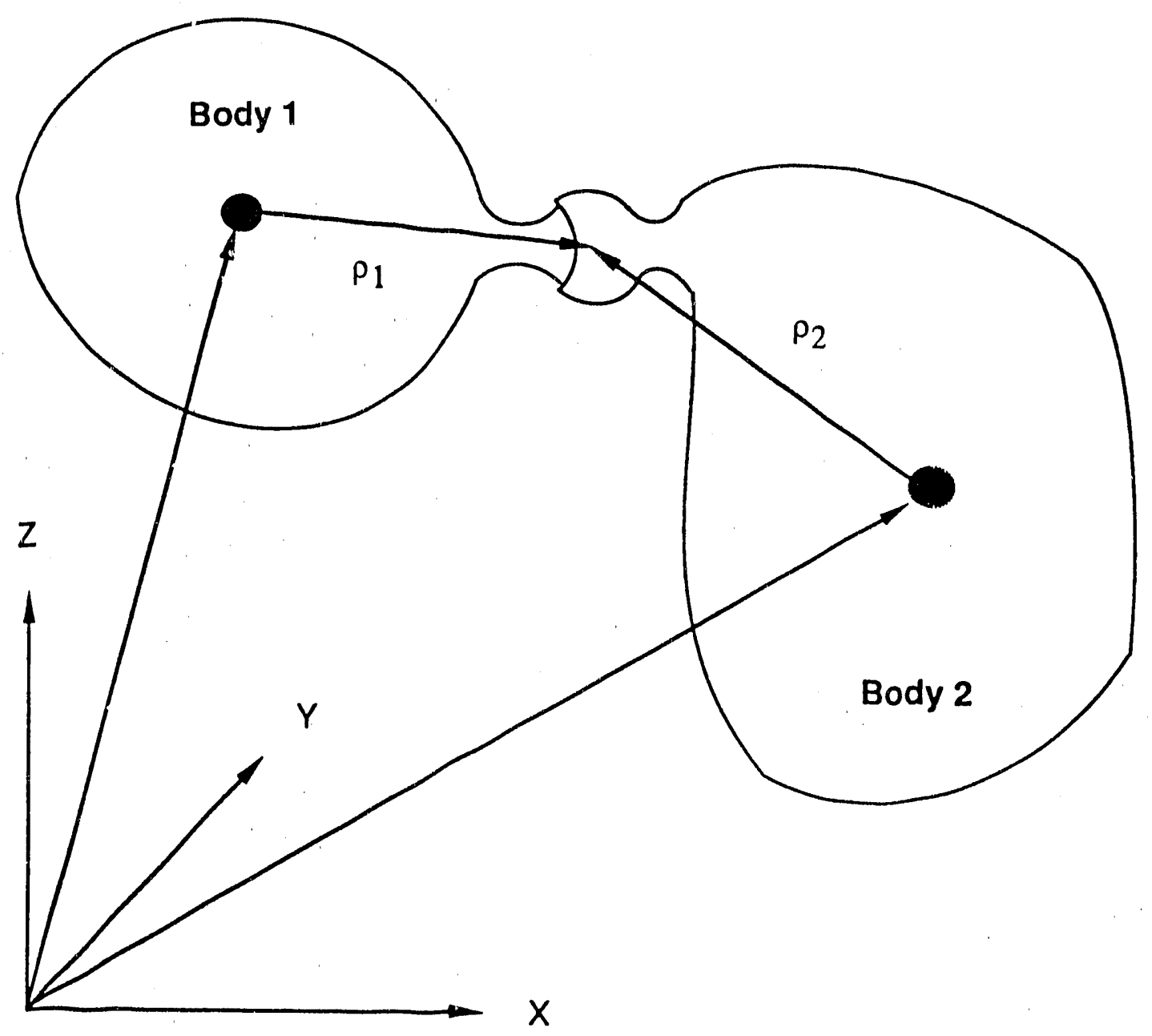

Figure 3: Spherical Joint 


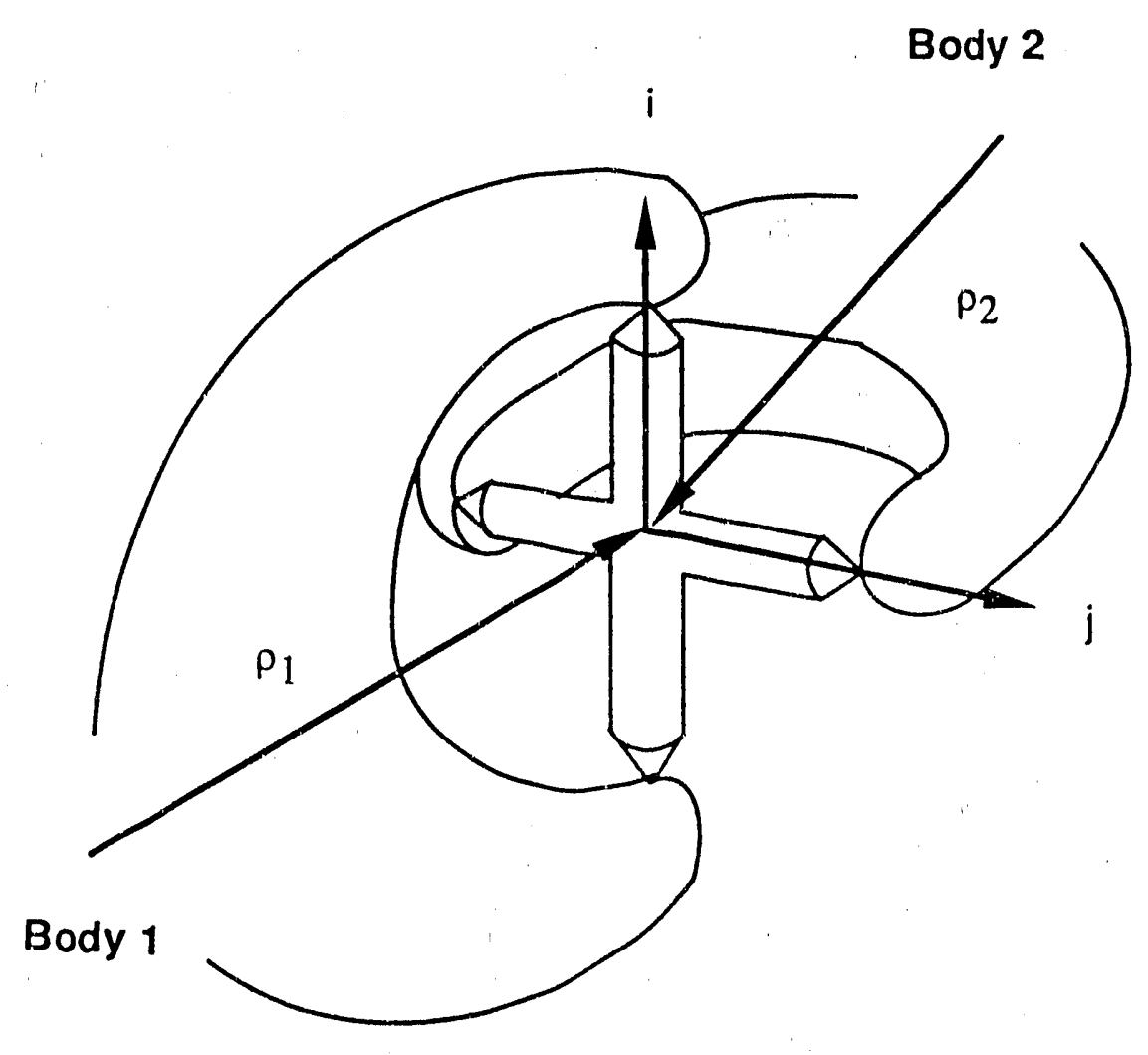

Figure 4: Universal Joint 


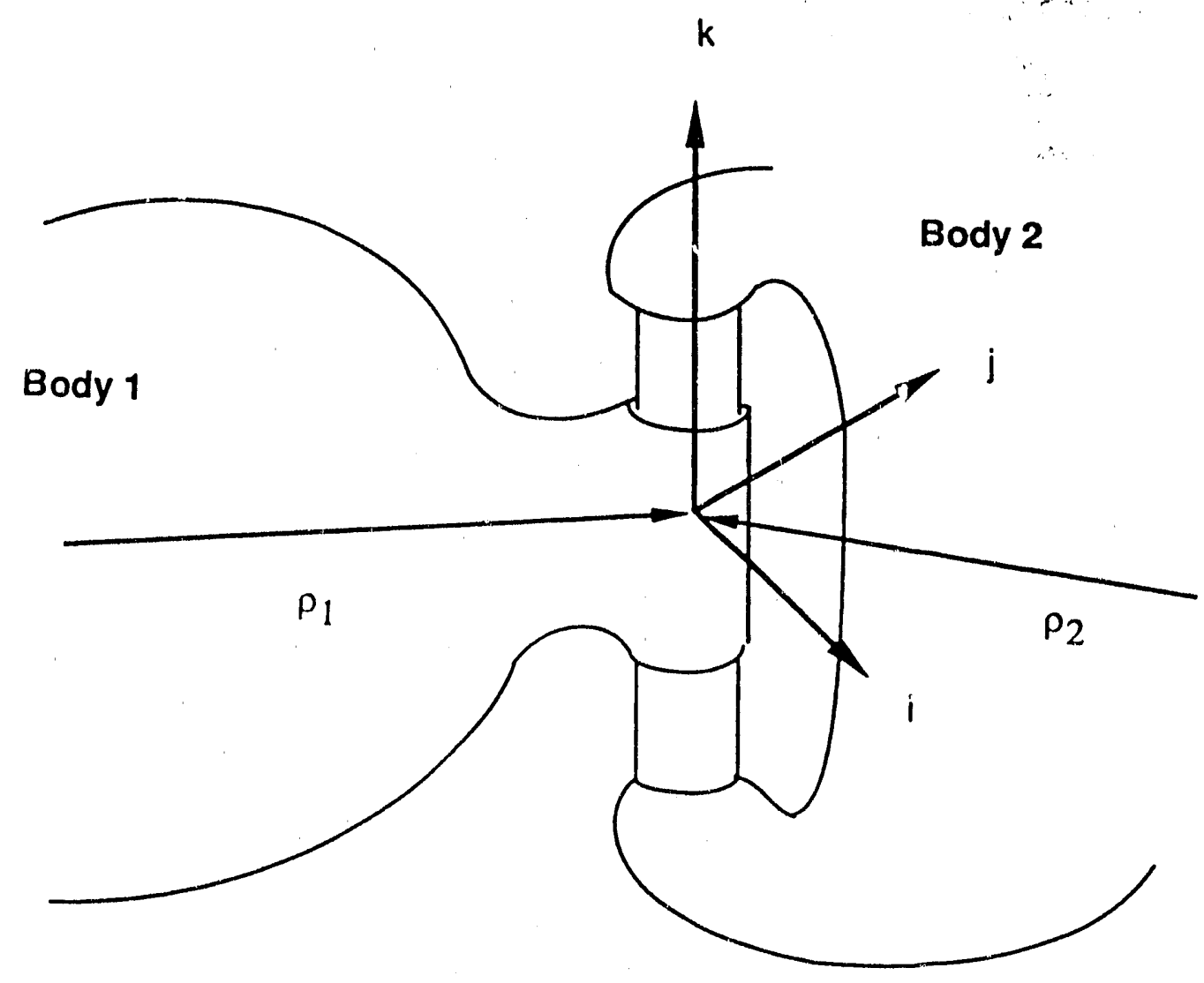

Figure 5: Revclute Joint 


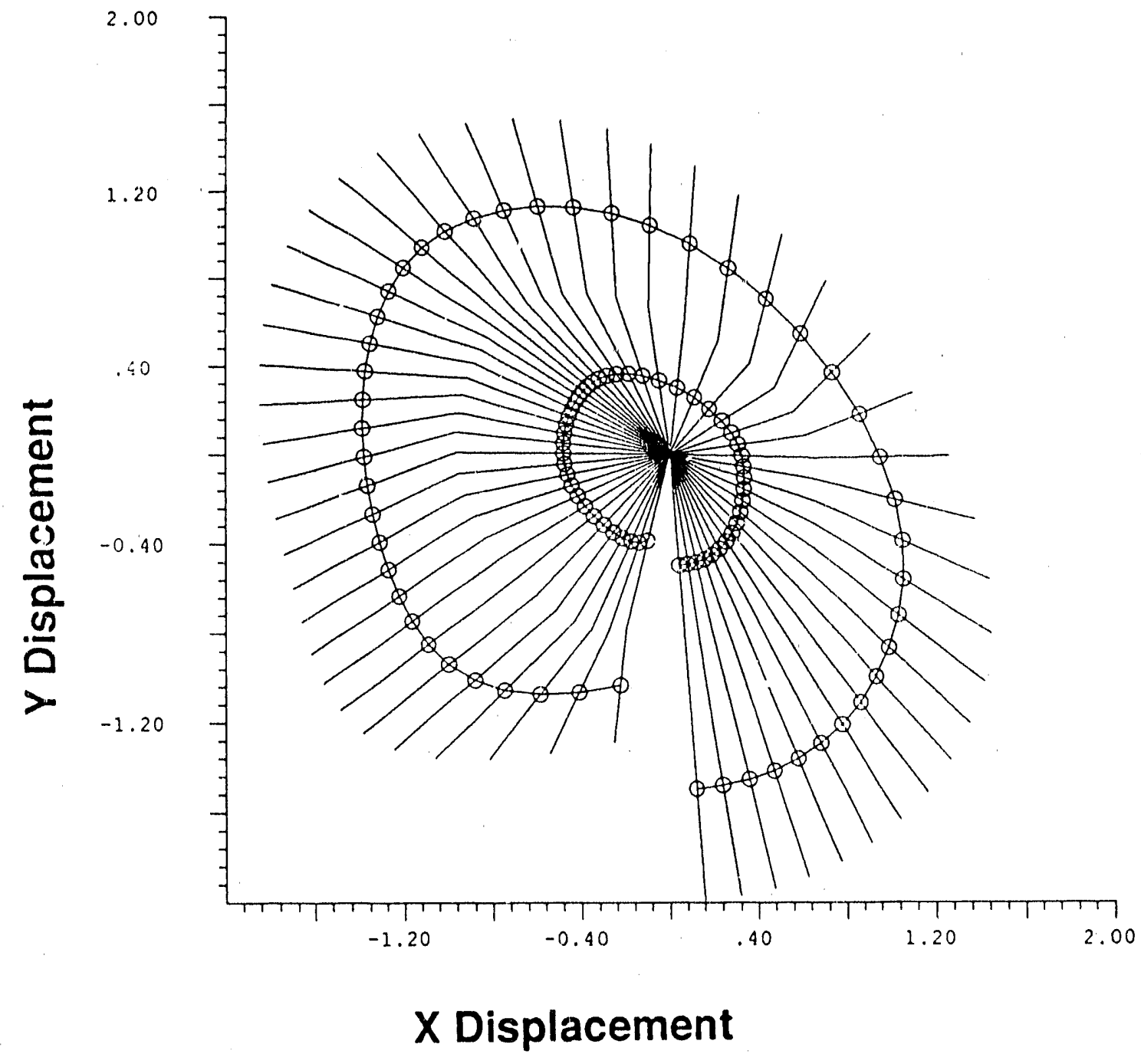

Figure 6: XY Displacement - Spherical Joint Case 1 


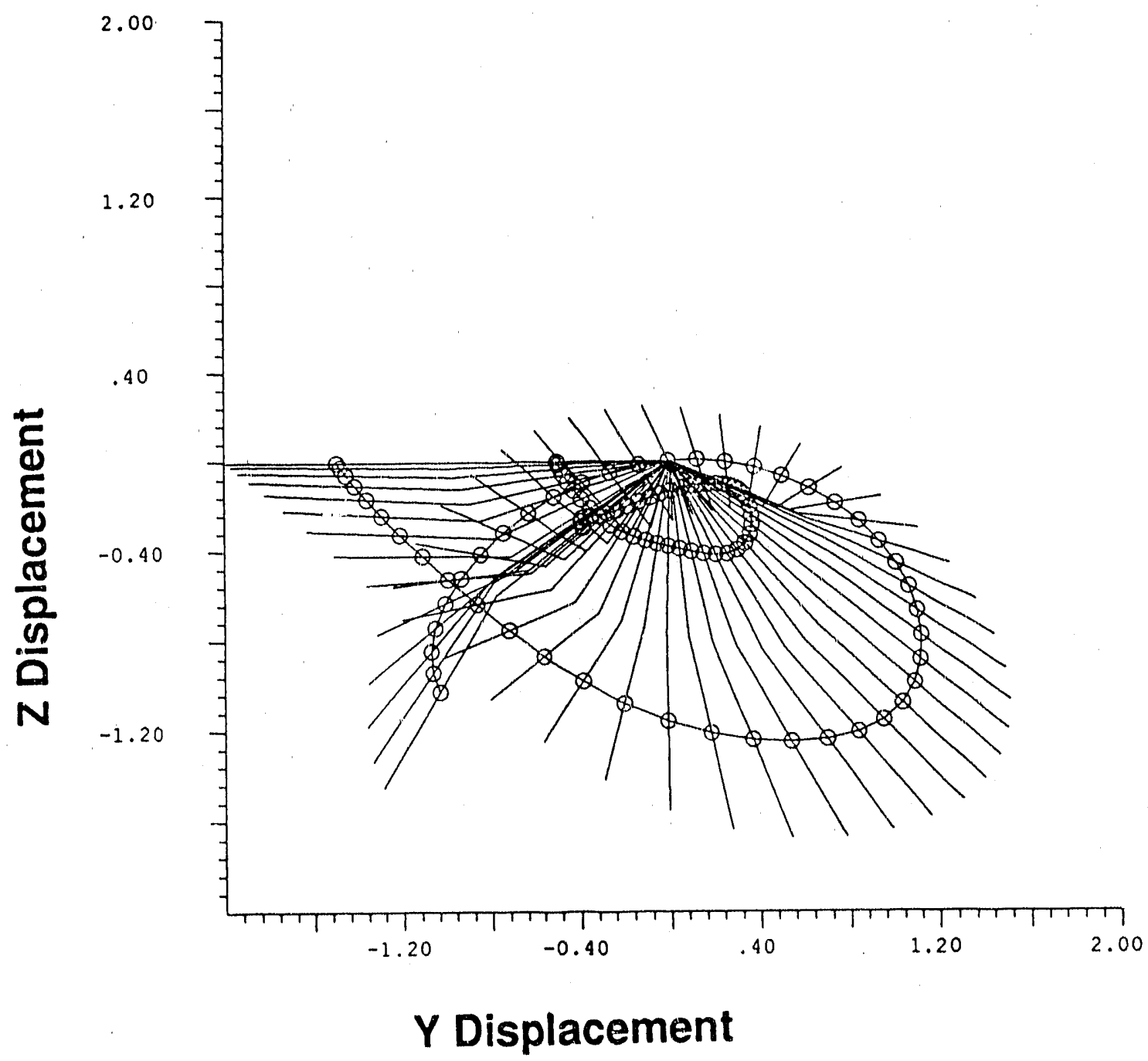

Figure 7: YZ Displacement - Spherical Joint Case 1 


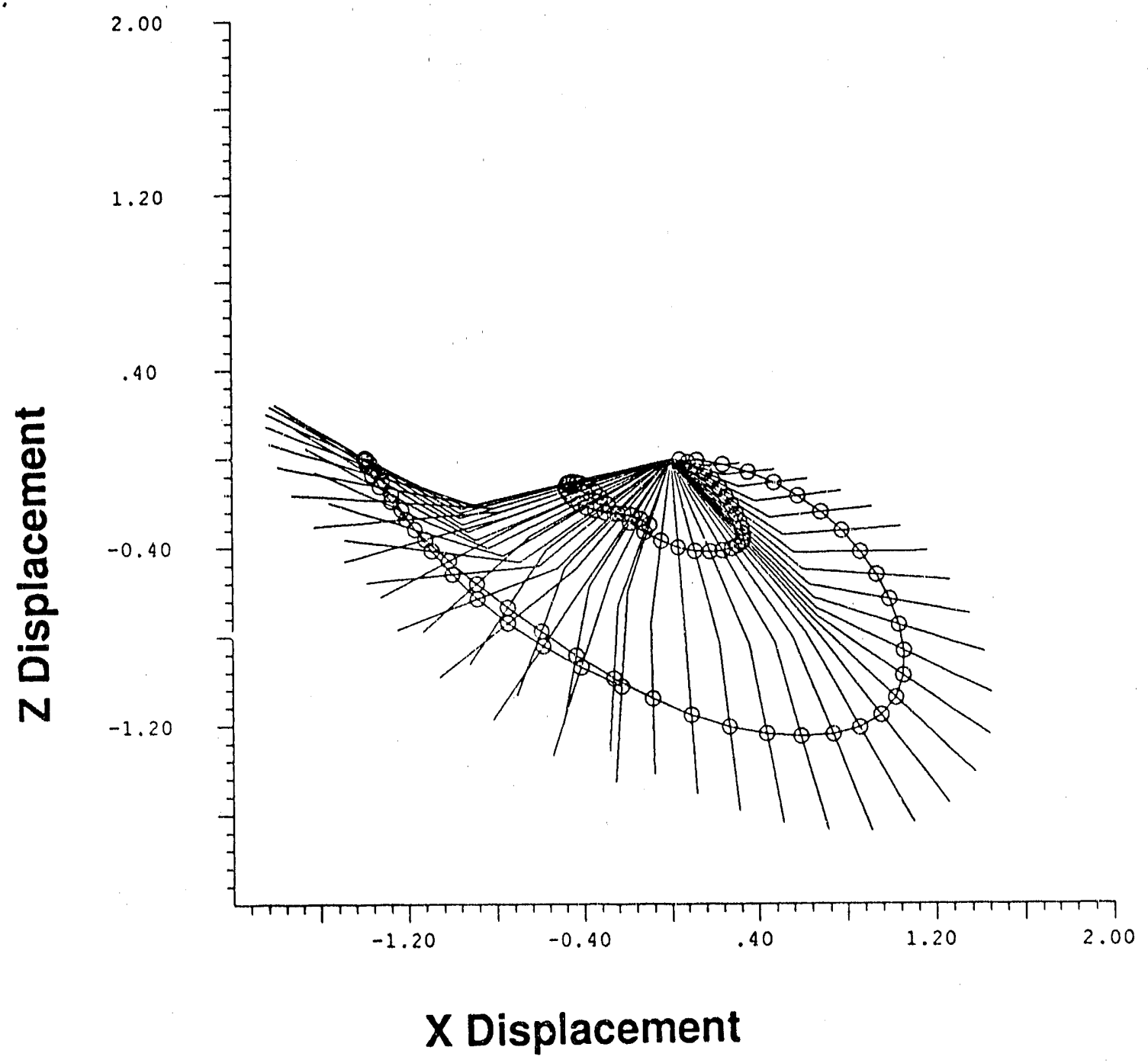

Figure 8: XZ Displacement - Spherical Joint Case 1 


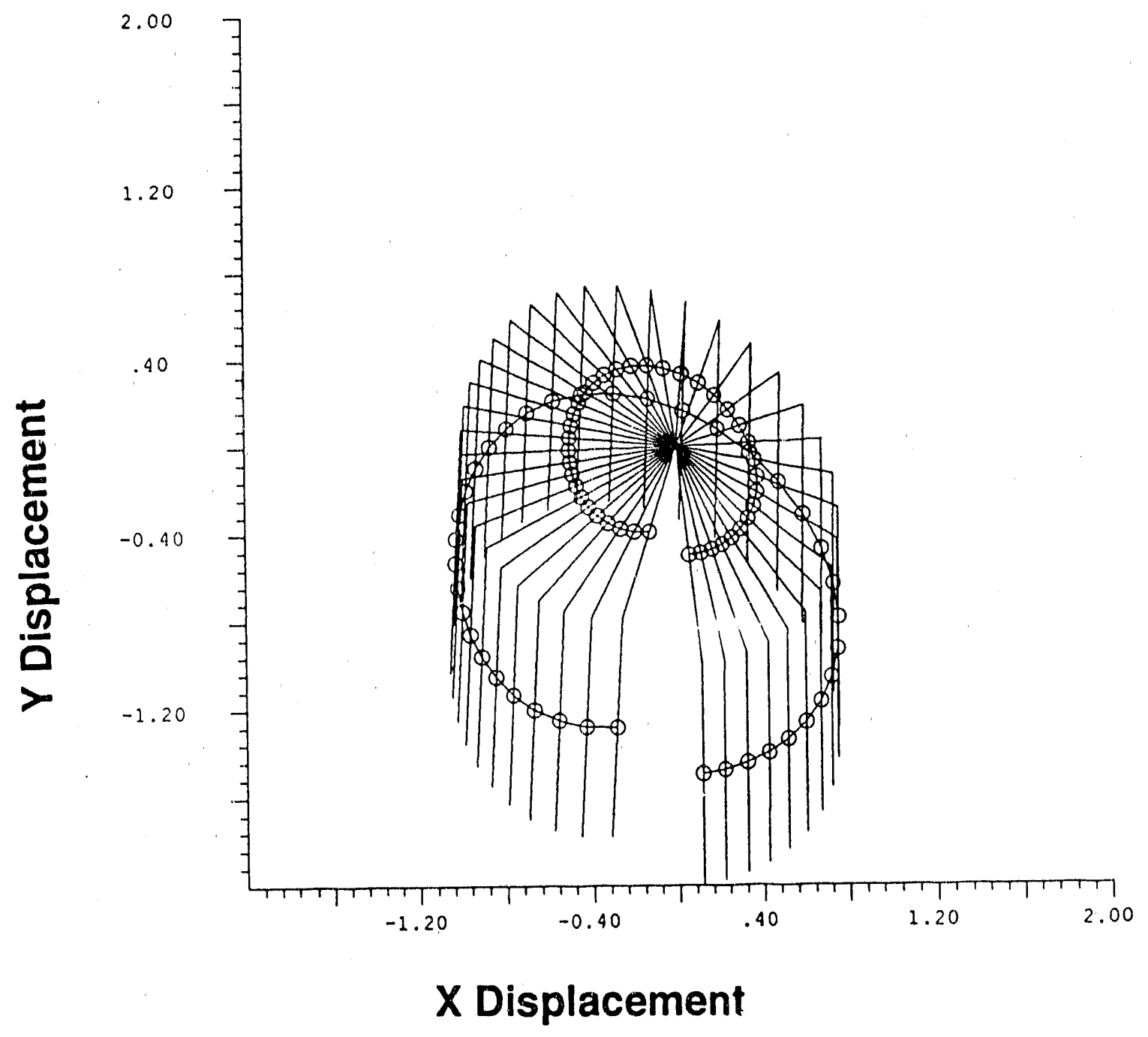

Figure 9: XY Displacement - Spherical Joint Case 2 


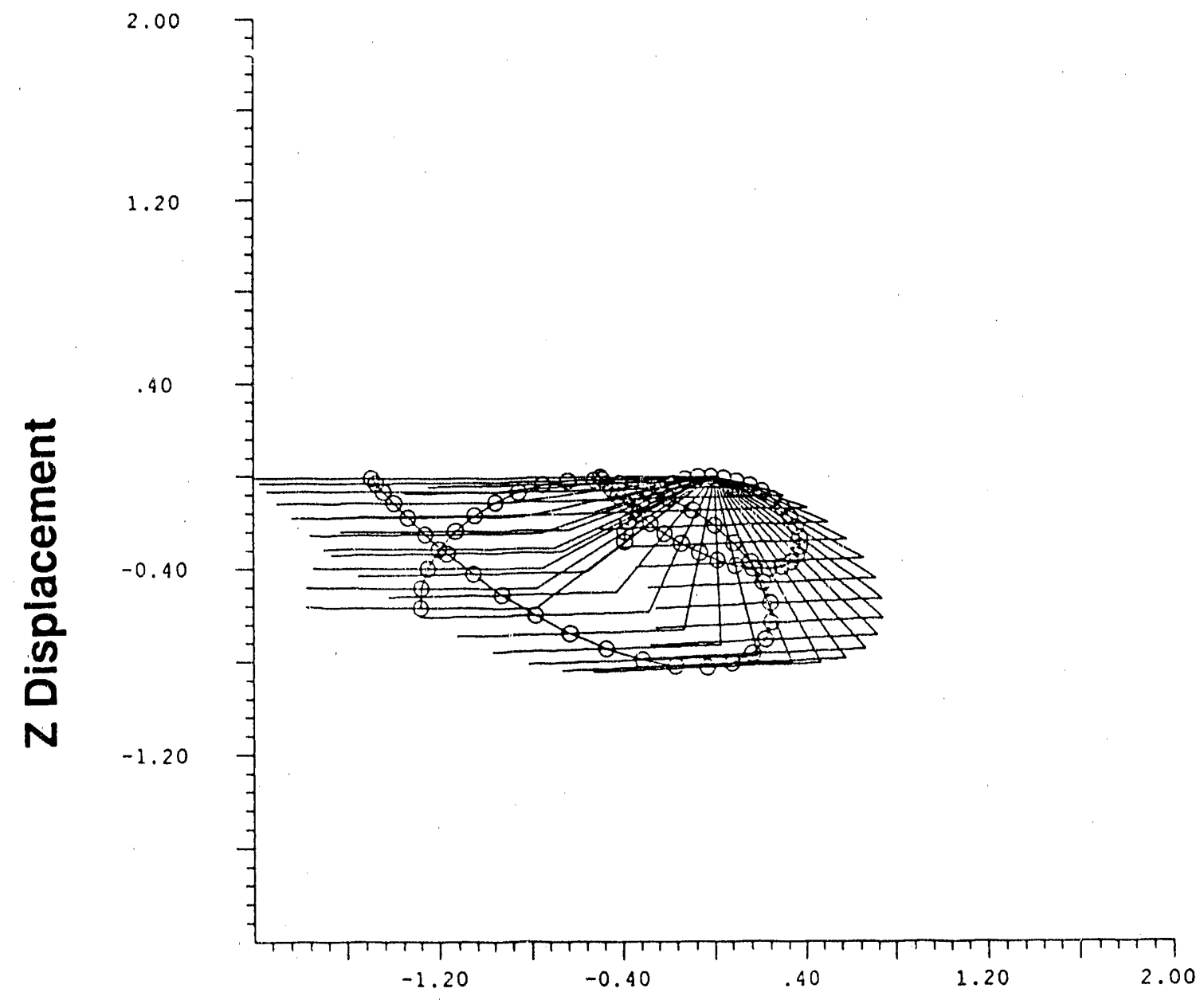

Y Displacement

Figura 10: YZ Displacement - Spherical Joint Case 2 


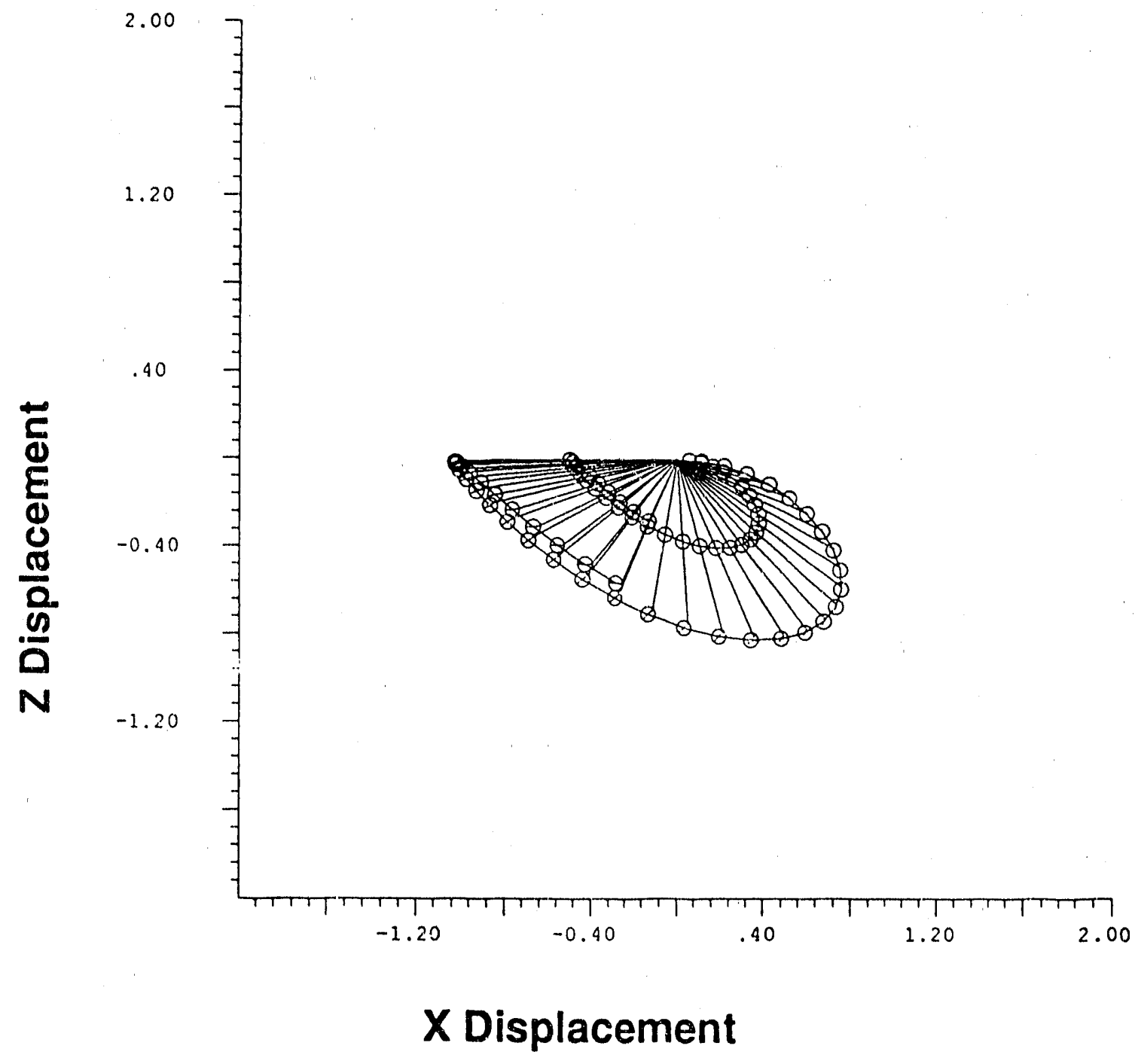

Figure 11: XZ Displacement - Spherical Joint Case 2 


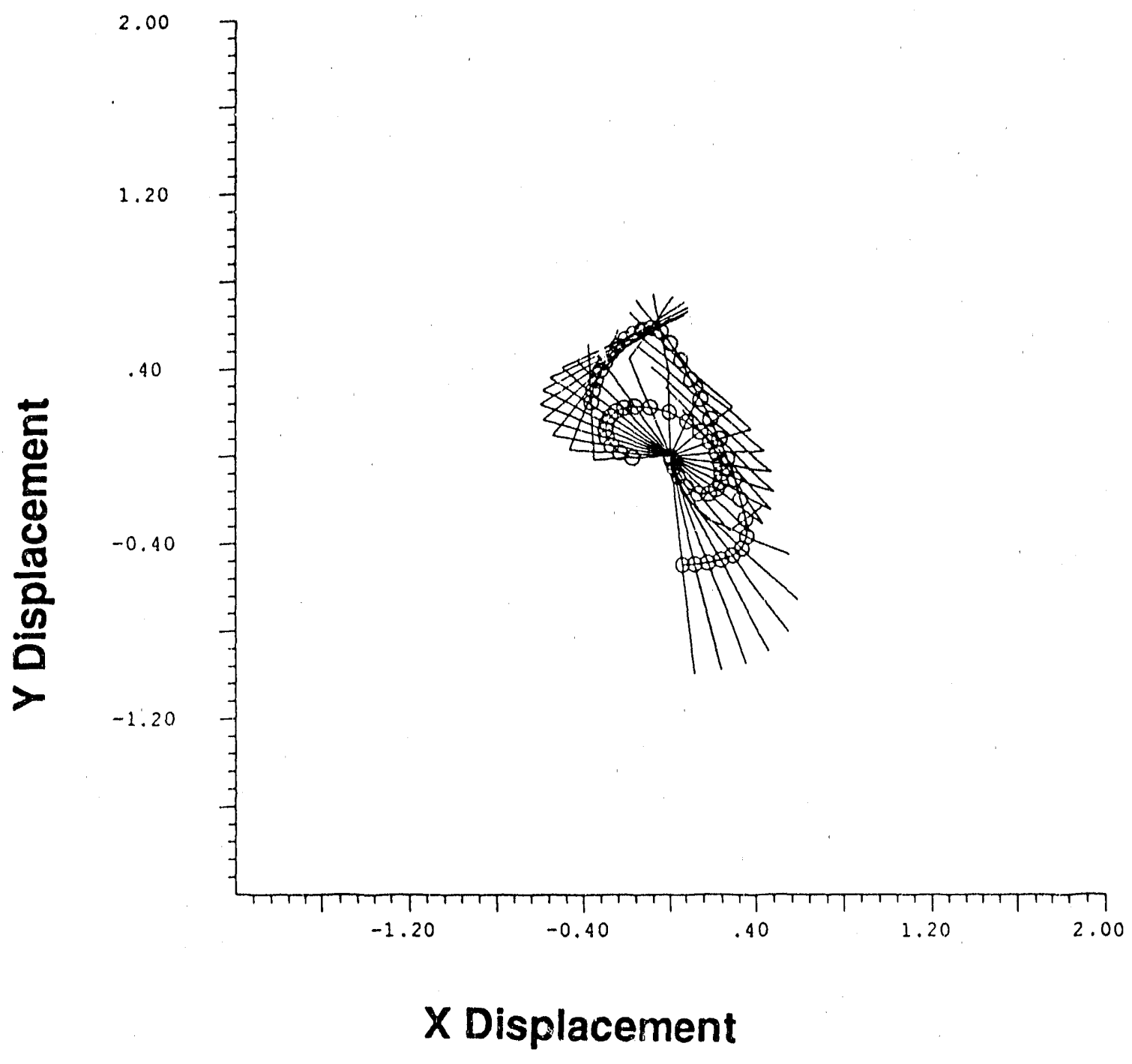

Figure 12: XY Displacement - Universal Joint 


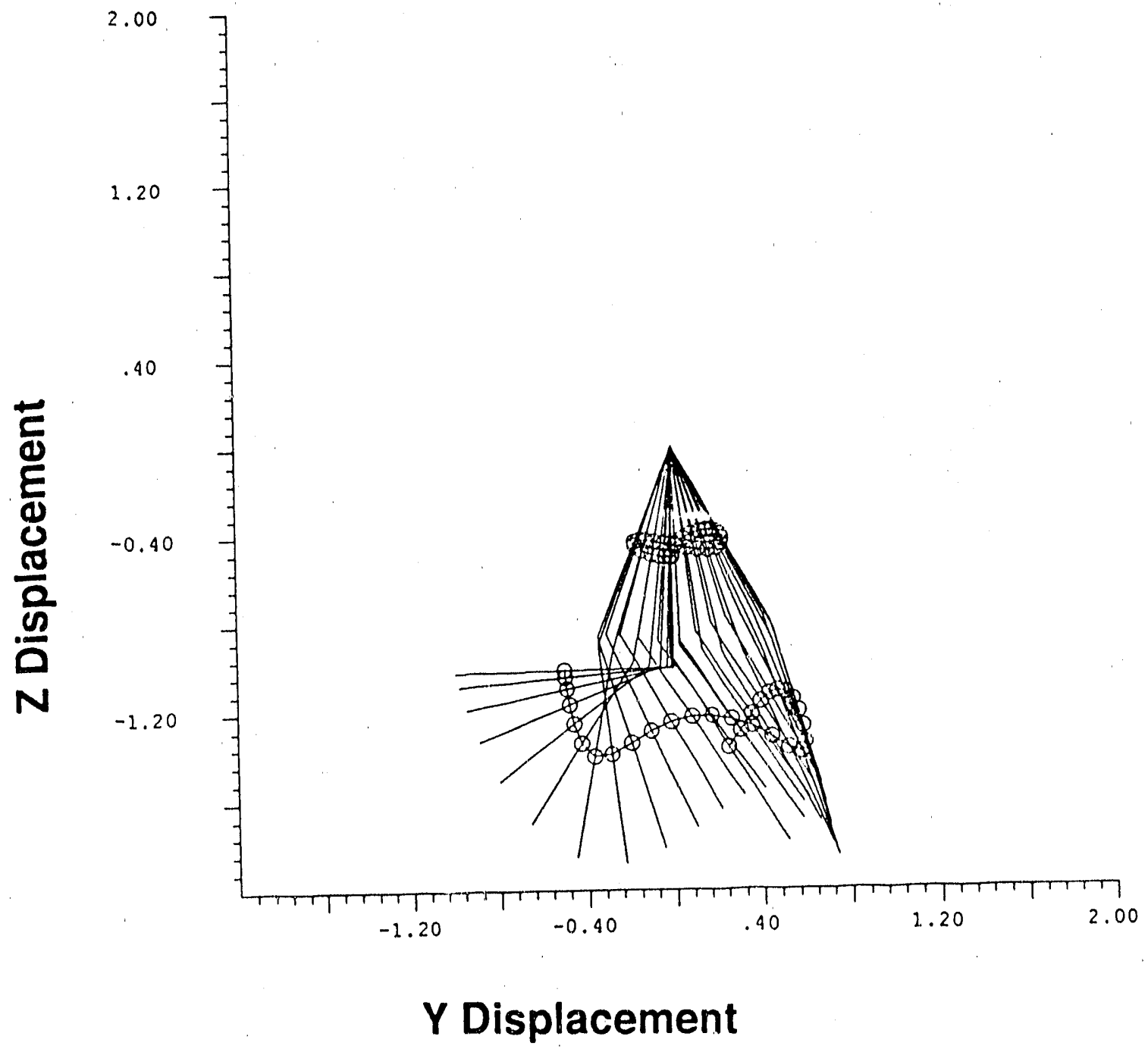

Figure 13: YZ Displacement - Universal Joint 


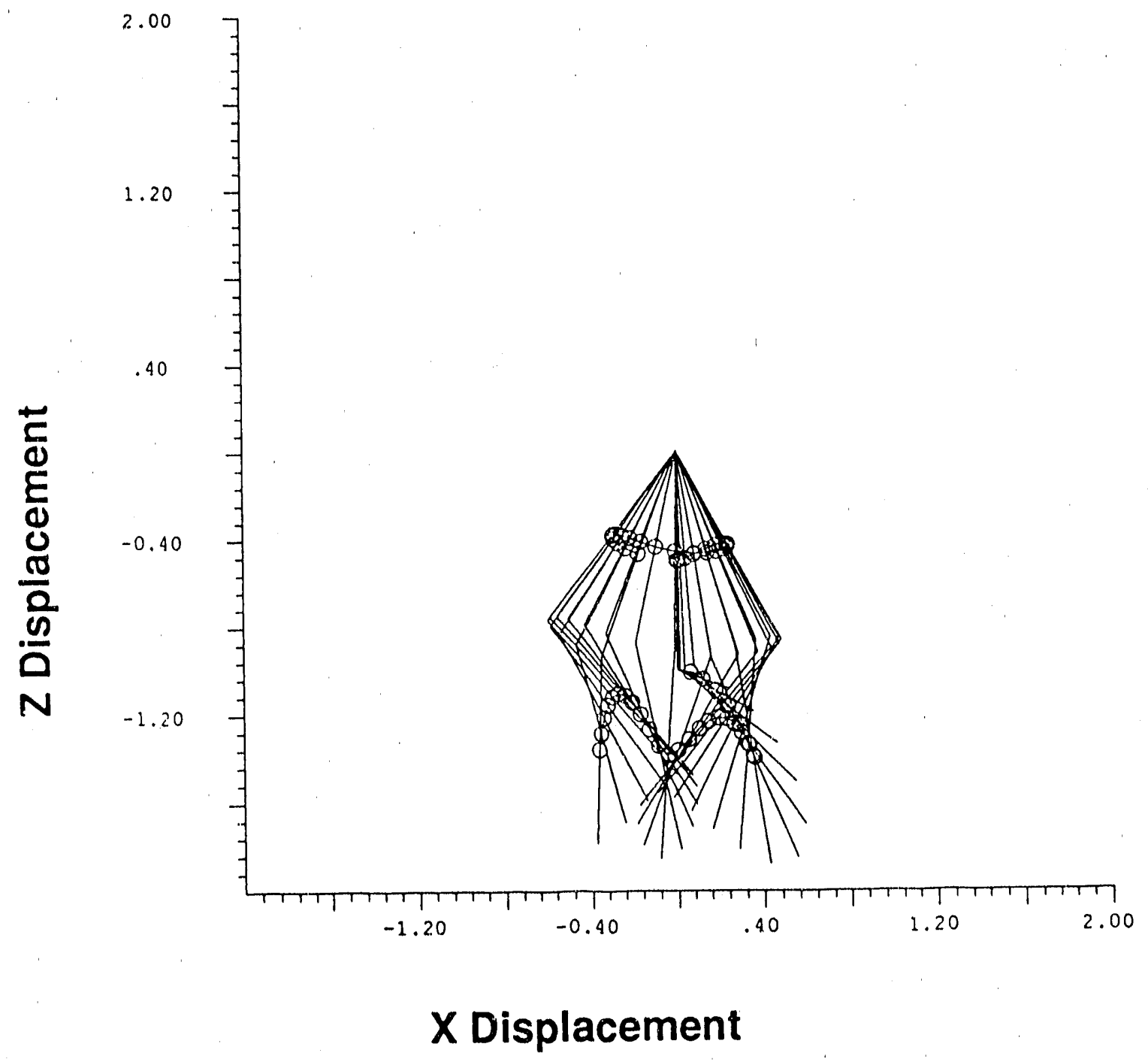

Figure 14: XZ Displacement - Universal Joint 


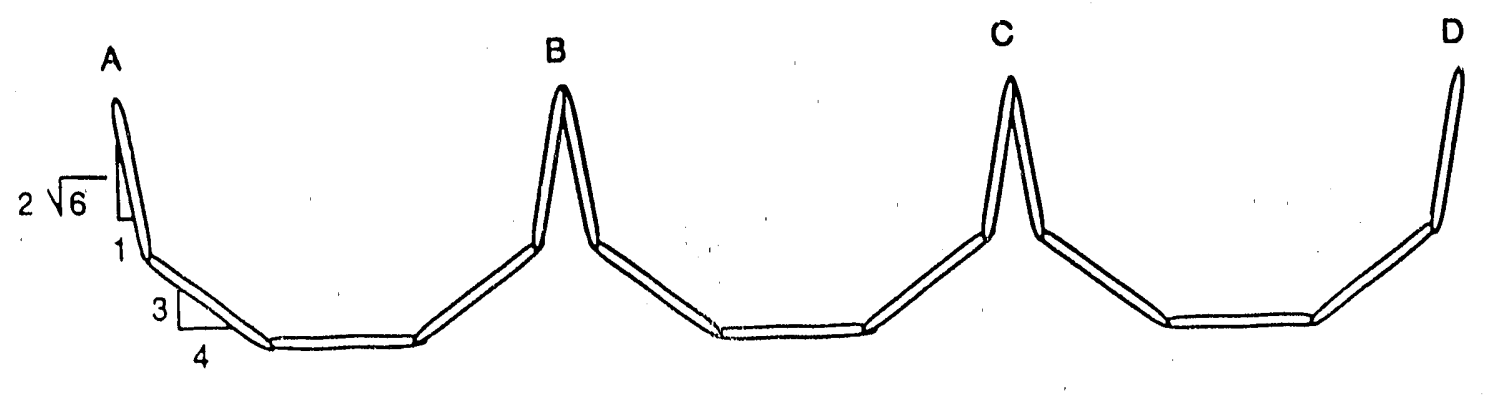

Figure 15: Fifteen Bar Linkage 


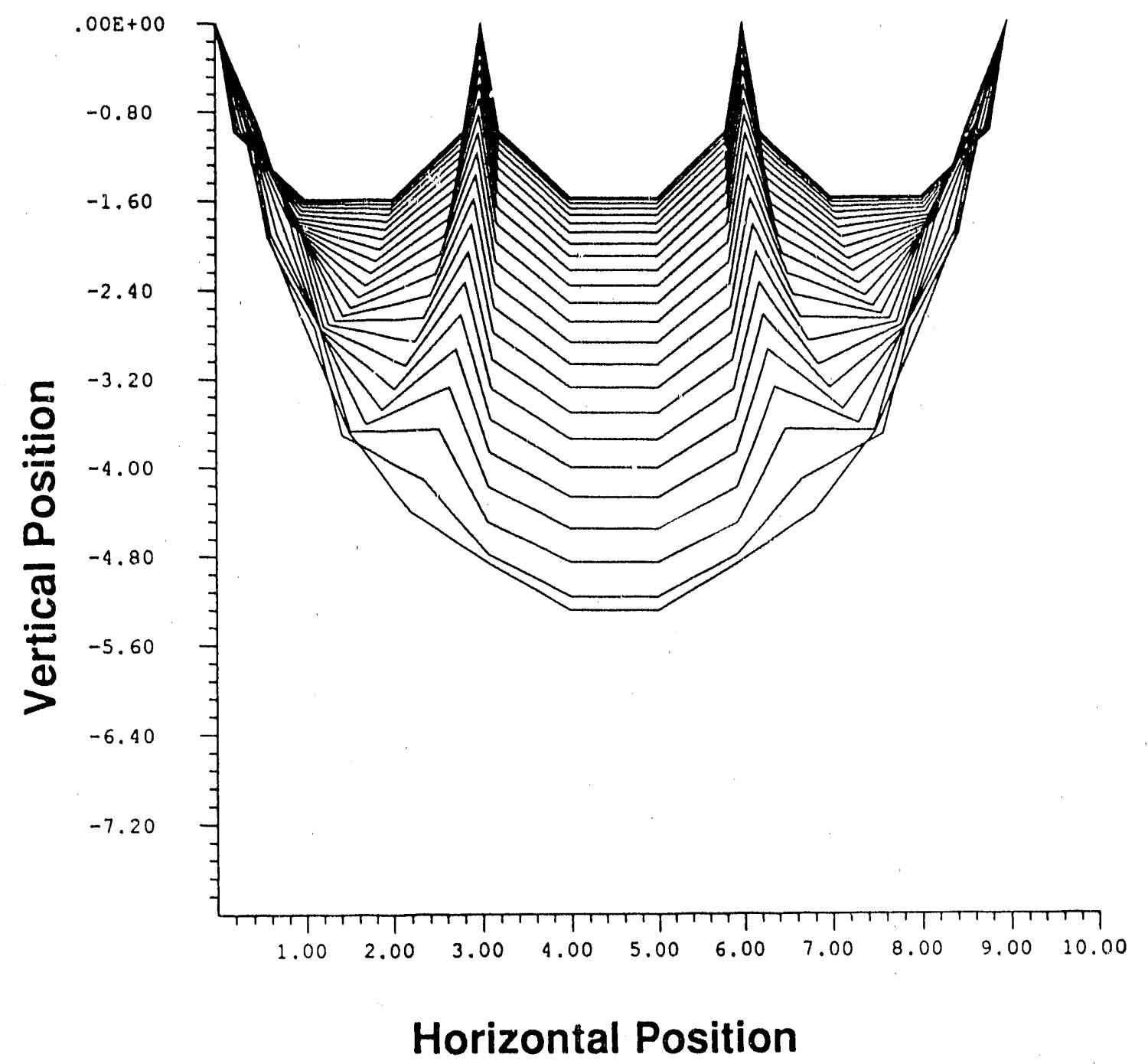

Figure 16: Linkage Profile - Falling 


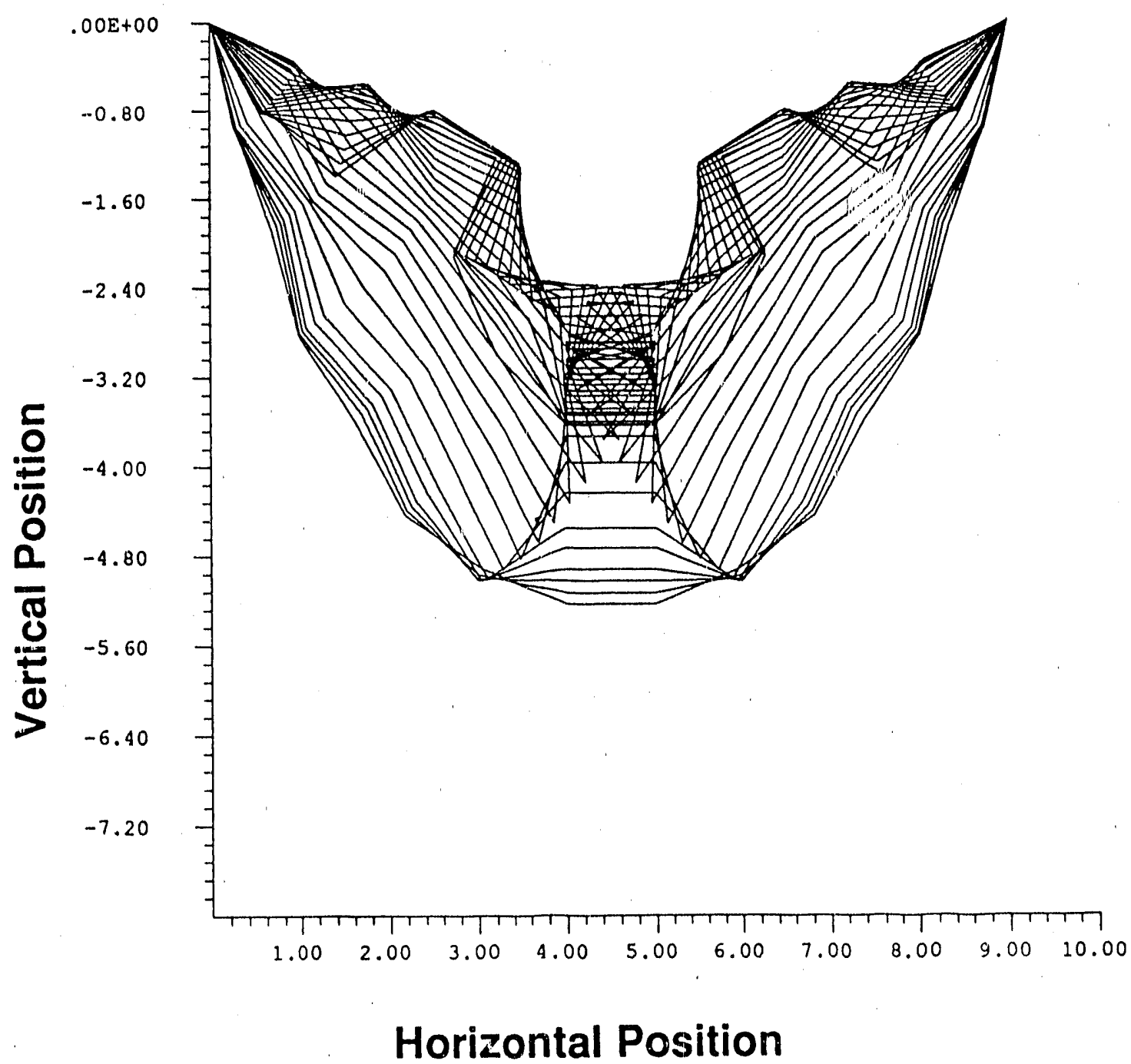

Figure 17: Linkage Profile - Rebounding 


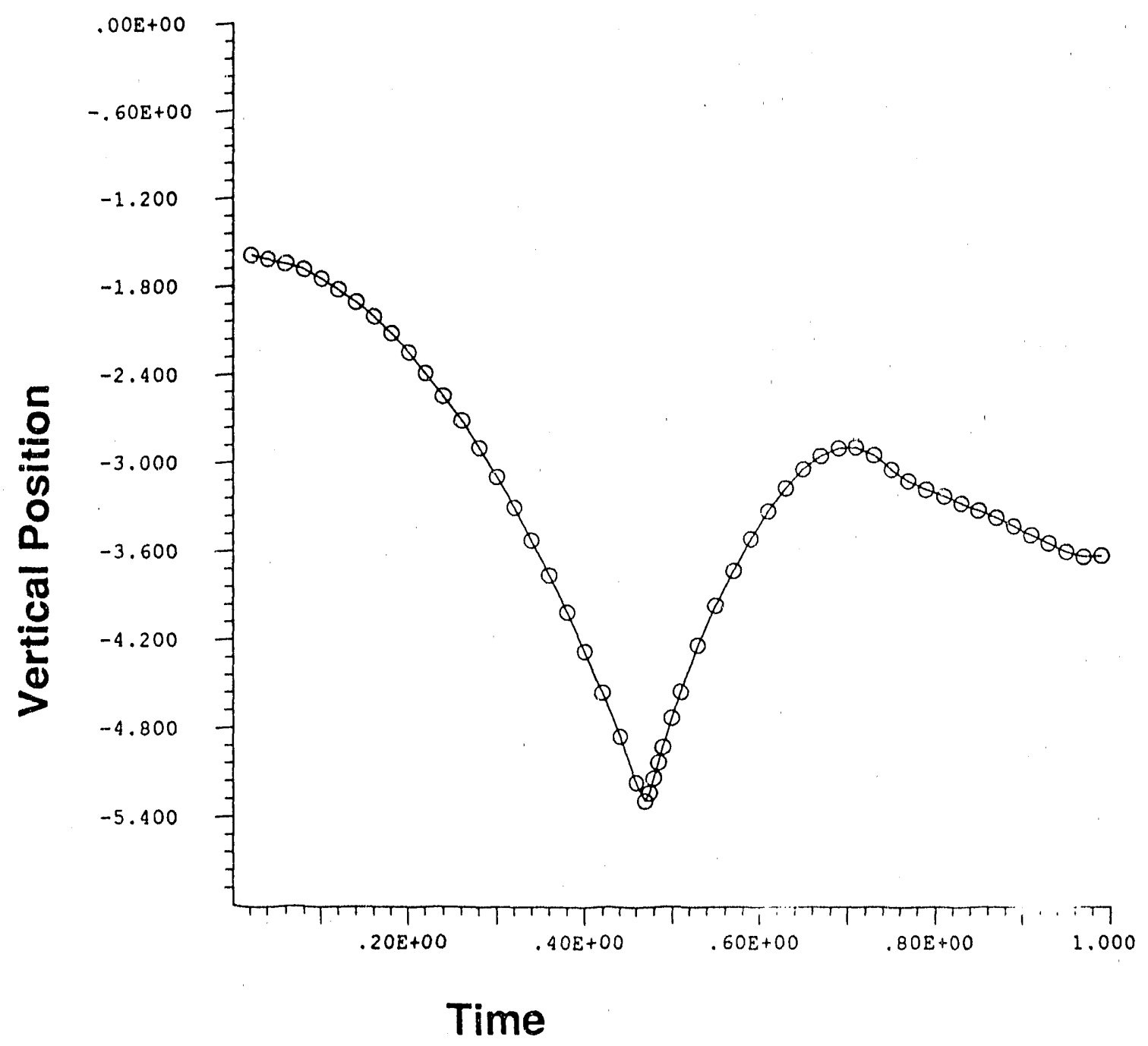

Figure 18: Vertical Displacement of Center Link 

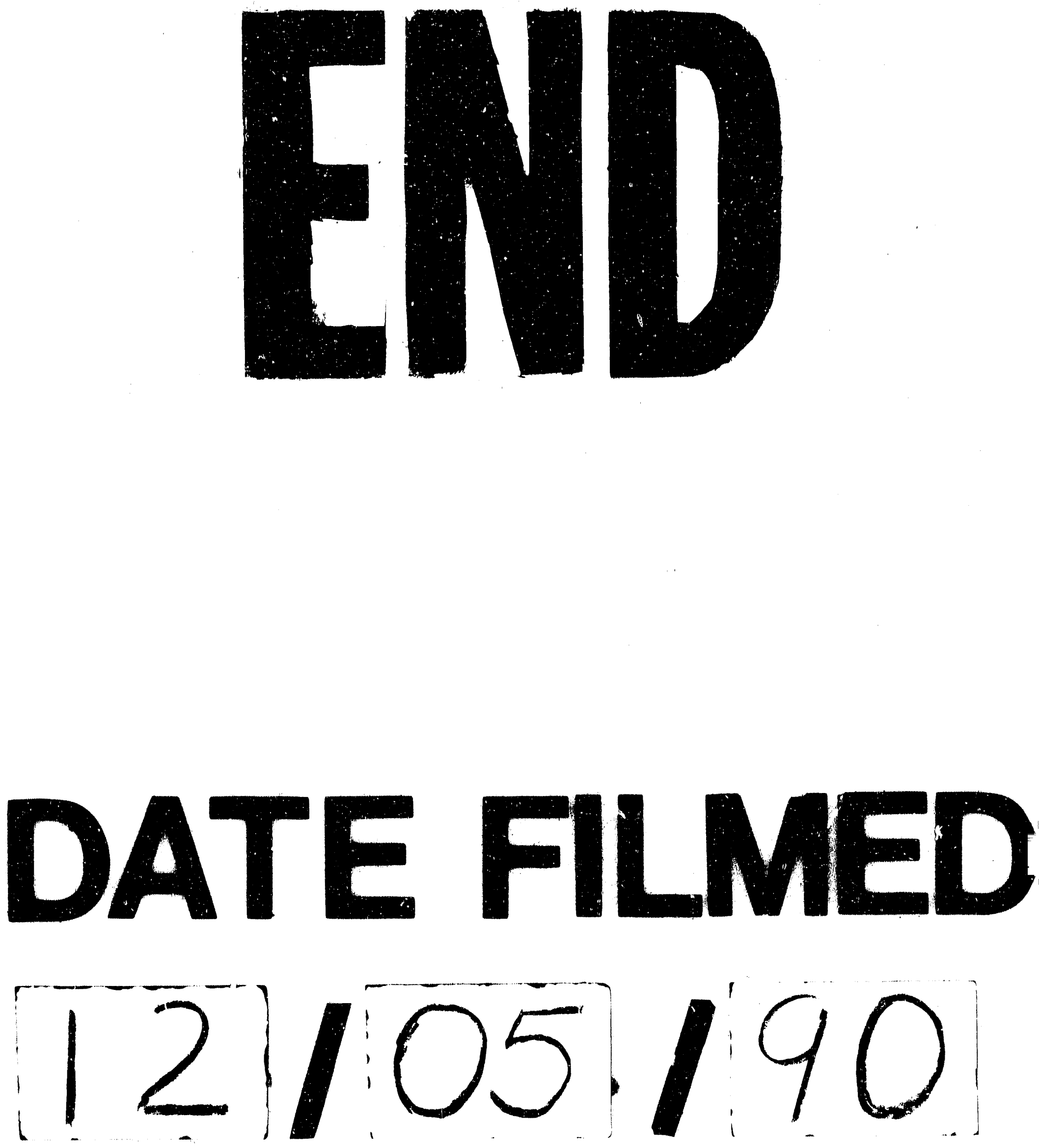
